This is the final peer-reviewed accepted manuscript of:

Catellani, C., Bianchi, G., Falfari, S., Cazzoli, G. et al., "Assessment of Advanced SGS Models for LES Analysis of ICE Wall-Bounded Flows - Part I: Basic Test Case" SAE Int. J. Engines 9(1):657-673, 2016

The final published version is available online at: https://doi.org/10.4271/2016-019041

Rights / License:

The terms and conditions for the reuse of this version of the manuscript are specified in the publishing policy. For all terms of use and more information see the publisher's website.

This item was downloaded from IRIS Università di Bologna (https://cris.unibo.it/)

When citing, please refer to the published version. 


\title{
ASSESSMENT OF AdVANCED SGS MOdels FOR LES ANALYSIS OF ICE WALL-BOUNDED Flows - PART I: BASIC TEST CASE
}

A PREPRINT OF DOI:10.4271/2016-01-9041

\author{
Cristian Catellani, Gian Marco Bianchi, Stefania Falfari, Giulio Cazzoli, Claudio Forte \\ University of Bologna
}

February 12, 2020

\begin{abstract}
Large Eddy Simulation (LES) represents nowadays one of the most promising techniques for the evaluation of the dynamics and evolution of turbulent structures characterizing internal combustion engines (ICE). In the present paper, subdivided into two parts, the capabilities of the open-source CFD code OpenFOAM ${ }^{\mathbb{R}}$ v2.3.0 are assessed in order to evaluate its suitability for engine cold flow LES analyses. Firstly, the code dissipative attitude is evaluated through an inviscid vortex convection test to ensure that the levels of numerical dissipation are compatible with LES needs. Quality and completeness estimators for LES simulations are then proposed. In particular the Pope M parameter is used as a LES completeness indicator while the LSR parameter provides useful insights far calibrating the grid density. Other parameters such as the two-grid $L E S_{I Q k}$ index are also discussed. Then advanced SGS models such as the dynamic WALE and the Sigma models are compared with models traditionally used for LES simulations of ICE wall-bounded flows, i.e. the dynamic Smagorinsky and the WALE models. The proposed SGS models have been implemented in the open-source CFD code OpenFOAM ${ }^{\mathbb{R}}$, extending its standard capabilities. Validation of the implemented models has been performed using two different test cases: the Dellenback abrupt expansion, which is presented in part I, and the Thobois stationary flow bench, presented in part II. The results have been evaluated through comparison with experimental data and the completeness of the LES simulations has been assessed using the aforementioned quality estimators.
\end{abstract}

\section{CITATION:}

Catellani, C., Bianchi, G., Falfari, S., Cazzoli, G. et al., "Assessment of Advanced SGS Models for LES Analysis of ICE Wall-Bounded Flows - Part I: Basic Test Case," SAE Int. J. Engines 9(1):2016, doi:10.4271/2016-01-9041. 2016-01-9041 Published 03/14/2016 Copyright (C) 2016 SAE International. doi:10.4271/2016-01-9041

\section{INTRODUCTION}

It is nowadays agreed that LES models will be the next generation of turbulence models for several CFD applications. This is particularly true in the field of ICE where the traditional RANS is the actual standard approach. In fact, one of the major issues with RANS modeling is its intrinsic inability to predict the unsteady flow structures characterizing ICE flows and which strongly affects complex phenomena like fuel-air mixing and cycle-to-cycle engine variability. With the advent of new engine concepts like GDI, HCCI etc. and with the continuously increasing severity of the legislation regarding pollutant emission limitations and engine efficiency, the adoption of more accurate predictive models is not an option anymore and here is where LES could make the difference over RANS.

In the RANS approach the Navier-Stokes equations are time averaged, therefore loosing details on the flow turbulent fluctuations. In the LES methodology the aforementioned equations are filtered in space, directly resolving the larger scales of motion, while the smaller scales are modeled through Sub-Grid Scale (SGS) models. In this way LES provides a more physical representation of the turbulent structures being capable of reproducing the most of the unsteady flow features characterizing turbulent flows. 
The continuous increase in computing power availability of the last decades has contributed to the growing interest in LES technique despite its huge computational demands when compared to RANS. This is true not only for academic research but also for industry, where LES could be a promising investigation tool for several engineering applications, amongst which ICE configurations.

The first LES applications to ICE flows dealt with simple stationary configurations: in [1] and [2] Thobois performed a systematic assessment of the LES methodology applied to a steady flow bench. The same case has been later analyzed by Brusiani [3]-[4] and Piscaglia [5], focusing on the assessment of LES quality. In [6] and [7] Keskinen tackled more realistic engine configurations demonstrating the capabilities of LES in reproducing the unsteady complex behavior of ICE flows. Realistic engine geometries have been studied by Huijnen in [8] in which the Authors have shown the superior capabilities of LES over URANS approach in resolving the inhomogeneous features of turbulence.

Other studies dealt with non-stationary cases such as the one proposed by Moureau [9] in which LES was used to study the generation and disruption of compressed tumble vortexes inside a square piston engine. Further works [10], [11], [12], [13] have demonstrated the capabilities of LES in retrieving information about cycle-to-cycle engine variability. In a few studies [14], [15], [16] the Authors modeled also the combustion process obtaining detailed descriptions of realistic IC engine flows.

Despite the growing number of LES applications to IC engine simulation, there are, still today, some open modeling issues that must be considered.

Firstly, it must be kept in mind that for a LES simulation to be considered as such, the fraction of the resolved turbulent kinetic energy must be greater than $70-80 \%$ of the total turbulent kinetic energy, as highlighted by Pope in [17]. An insufficient resolution would in fact mean that the simulation is modeling a too wide fraction of the turbulent spectrum degrading the statistical characterization of the flow. To date, few Authors have taken into consideration this issue such as Piscaglia [5], Celik [18], Di Mare [19] or Brusiani [20], this latter also proposing a LES quality parameter estimator.

A second issue related to the former one concerns the numerical solution of the (filtered) Navier Stokes equations on unstructured grids, typical for IC engine applications. It is well known by literature [21] that such process tends to introduce numerical dissipation and dispersion in the solution. It is therefore of paramount importance to assess the solver dissipative behavior, evaluating its suitability for LES simulations.

Then several other aspects are important for the practicability of LES of realistic IC engine configurations. The discretization of the computational domain plays an important role in defining the filter width of (implicitly filtered) LES. In this regard the element type is an issue of major interest: despite the common acknowledgement that hexahedral elements provide less numerical diffusivity, some Authors [3], [11], [22] have successfully performed LES of ICErelated flows employing tetrahedral elements. These type of elements, among with polyhedral ones, are certainly more prone to adapt to the complexity of realistic engine geometries.

Another topic of interest is the near-wall treatment: it is well known that for the sake of LES completeness the resolution at the walls should approach $y^{+} \approx 1$. This requirement is generally unfeasible for realistic cases due to the high computational costs. Some Authors like Brusiani [3], Abraham [23] and [24], have demonstrated the feasibility of near-wall modeling (i.e. the use of wall functions) for ICE cases where the focus is primarily in resolving the turbulent fluctuations in the core flow.

Again, numerical schemes and boundary condition types are active research fields. For the former there is a growing interest in higher order methods ([25]) with the pursue of an increase in both space and time resolution accuracy. However, it must be noted that nowadays most of the ICE LES applications are based on second order schemes due to their greater robustness and reliability. Then, boundary conditions in LES should be able to provide a suitable treatment for turbulence properties at domain inlets and to allow acoustic waves to properly leave the domain without reflection.

Finally SGS modeling represents a further difficulty when simulating ICE flows. They not only have to correctly model the effects of unresolved residual fluid motion but must also guarantee the proper near-wall eddy-viscosity behavior, in particular when coarse grids are adopted. Several attempts to overcome this issue have been carried out, such as the WALE model proposed by Nicoud et. al. [22] as an alternative to the classical Smagorinsky SGS model or the more commonly adopted dynamic version.

The present paper addresses the use of advanced SGS models for LES of IC engine flows. Such models, namely the "dynamic Wale" and "Sigma", proposed by Baya Toda et al. [26]-[27], are intended to be advancement of the Wale and dynamic Smagorinsky models that are today the most common choice for the aforementioned cases. 
This paper is divided into three main sections. In the first one some theoretical aspects of LES modeling are recalled together with the details of the SGS models implemented in the open-source CFD code used for the present work [28][29]. Quality criteria for LES simulations will also be presented and discussed. In the second section the capabilities of the CFD solver in terms of level of numerical dissipation and dispersion will be assessed. This will allow to test the suitability of the code for LES analyses and will provide some guidelines for an optimal choice of discretization and numerical schemes. In the latter section of the paper the Dellenback abrupt expansion test case [30] will be used to evaluate the implemented SGS models. The results will be compared against both experimental data and numerical simulations run with "standard" SGS models. Quality assessment of the analyses will also be presented and discussed.

\section{LES MODELLING}

\section{The Filtered Navier-Stokes Equations}

The governing equations describing the motion of incompressible fluids are the Navier-Stokes (NS) equations in their incompressible formulation. In LES such equations are spatially filtered in order to retain only the larger scales of motion. Using an overbar to denote the filtering operation and adopting the Einstain notation, the filtered NS equations read:

$$
\frac{\partial \bar{u}_{i}}{\partial t}+\frac{\partial\left(\bar{u}_{i} \bar{u}_{j}\right)}{\partial x_{j}}=-\frac{1}{\rho} \frac{\partial \bar{p}}{\partial x_{i}}+\nu \frac{\partial^{2} \bar{u}_{i}}{\partial x_{j} \partial x_{j}}-\frac{\partial \tau_{i j}^{S G S}}{\partial x_{j}}
$$

and the fluid incompressibility constrain is expressed by:

$$
\frac{\partial \bar{u}_{i}}{\partial x_{i}}=0
$$

where $u, p, r h o$ and $n u$ are respectively the fluid velocity, pressure, density and (molecular) kinematic viscosity. The filtered equation set 1-2 are formally similar to the original NS equations except for the term containing the sub-grid stresses defined as:

$$
\tau_{i j}^{S G S}=\overline{u_{i} u_{j}}-\bar{u}_{i} \bar{u}_{j}
$$

This term vanishes if the grid density is sufficiently high. In such case the filter width is small enough so that the LES tends to a DNS. Unfortunately $\tau_{i j}^{S G S}$ contains unknown terms that have to be modeled. Despite the lack of a universally accepted SGS turbulence model for LES, the eddy-viscosity assumption is, to date, the most common modeling approach. The SGS stress tensor $\tau_{i j}^{S G S}$ can be then expressed as:

$$
\tau_{i j}^{S G S}-\frac{1}{3} \tau_{k k}^{S G S} \delta_{i j}=-2 \nu_{S G S} \overline{S_{i j}}
$$

where $\overline{S_{i j}}$ is the resolved rate-of-strain tensor defined as:

$$
\overline{S_{i j}}=\frac{1}{2}\left(\frac{\partial \bar{u}_{i}}{\partial x_{j}}+\frac{\partial \bar{u}_{j}}{\partial x_{i}}\right)
$$

With the eddy-viscosity assumption, the formulation commonly adopted for $\nu_{S G S}$ is the following:

$$
\nu_{S G S}=\left(C_{m} \Delta\right)^{2} \overline{O P}(\vec{x}, t)
$$

where $C_{m}$ is the model constant, $\Delta$ is the filter width and $\overline{O P}$ is an operator specific of each sub-grid SGS model.

In the following paragraphs the basic details of the tested SGS models will be recalled. The dynamic Smagorinsky model was already present in the $O p e n F O A M^{\mathrm{R}}$ libraries but it has been modified in order to compute additional quantities of interest for the current investigation, such as $k_{S G S}$ or $\varepsilon_{S G S}$. All the other SGS models were not available in the libraries and they have been wholly implemented by the authors. 


\section{The Dynamic Smagorinsky SGS Model}

The dynamic Smagorinsky model is based on the dynamic procedure developed by Germano [31] and subsequent improvement proposed by Lilly [32]. The model employs a second filter, the test filter $\hat{\Delta}$ larger than the initial filter $\Delta$ and usually chosen as $\hat{\Delta}=2 \Delta$. The tensor operator $\overline{O P}$ in Eq. 6 is based on the second invariant of the strain rate tensor $\overline{S_{i j}}$ of the filtered velocity field $\overline{u_{i}}$ :

$$
\overline{O P}=\sqrt{2 \overline{S_{i j} S_{i j}}}
$$

The model constant is then dynamically evaluated from test-filtered resolved quantities:

$$
C_{m}^{2}=\frac{1}{2} \frac{<L_{i j} M_{i j}>^{+}}{<M_{i j} M_{i j}>}
$$

where $L_{i j}$ is the Leonard stress tensor:

$$
L_{i j}=\hat{u_{i} u_{j}}-\hat{\overline{u_{i}}} \hat{\overline{u_{j}}}
$$

and

$$
M_{i j}=\hat{\Delta}^{2} \hat{\overline{O P}} \hat{\overline{S_{i j}}}-\left(\Delta^{2} \widehat{\widehat{O P}} \overline{S_{i j}}\right)
$$

The superscript + in 8 means a positive clipping of all negative values to zero and the brackets $<>$ denote a volume averaging operation adopted in order to avoid numerical instability. To be noted that in the present work homogeneous volume averaging is employed.

\section{The WALE SGS Model}

The WALE model has been developed by Nicoud and Ducros [22] and its invariant is based on the square of the velocity gradient tensor. In particular, the operator $\overline{O P}$ is based on the traceless symmetric part of the square of the velocity gradient tensor $S_{i j}^{d}$, defined as

$$
S_{i j}^{d}=\frac{1}{2}\left({\overline{g_{i j}}}^{2}-{\overline{g_{j i}}}^{2}\right)-\frac{1}{3} \delta_{i j} \bar{g}_{k k}^{2}
$$

where $g_{i j}$ is the velocity gradient tensor. The tensor $S_{i j}^{d}$ can also be expressed as:

$$
\overline{S_{i j}^{d}}=\overline{S_{i k}} \overline{S_{k j}}+\Omega_{i k}^{-} \Omega_{k j}^{-}-\frac{1}{3} \delta_{i j}\left[S_{m n}^{-} S_{m n}^{-}-\Omega_{m n}^{-} \Omega_{m n}^{-}\right]
$$

being $\overline{S_{i j}}$ and $\overline{\Omega_{i j}}$ respectively the rate of strain and the vorticity tensors of the resolved velocity field. By construction the trace of $\overline{S_{i j}^{d}}$ is zero and its second invariant remains finite and proportional to $\overline{S_{i j}^{d}} \overline{S_{i j}^{d}}$. Assuming incompressibility, this latter term can be expressed as:

$$
\overline{S_{i j}^{d}} \overline{S_{i j}^{d}}=\frac{1}{6}\left(\overline{\mathbf{S}^{2}} \overline{\mathbf{S}^{2}}+\overline{\mathbf{\Omega}^{2}} \overline{\mathbf{\Omega}^{2}}\right)+\frac{2}{3} \overline{\mathbf{S}^{2}} \overline{\mathbf{\Omega}^{2}}+2 \mathbf{I} V_{S \Omega}
$$

where $\overline{\mathbf{S}^{2}}=\overline{S_{i j}} \overline{S_{i j}}, \overline{\Omega^{2}}=\overline{\Omega_{i j}} \overline{\Omega_{i j}}$ and $\mathbf{I} V_{S \Omega}=\overline{S_{i k}} \overline{S_{k j}} \overline{\Omega_{j l}} \overline{\Omega_{l i}}$.

Thanks to the operator $\overline{S_{i j}^{d}} \bar{S}_{i j}^{d}$ the WALE model is sensitive to both strain and rotation rates. The aforementioned operator is zero in case of pure shear and this means that unlike the Smagorinsky model, the WALE model avoids producing eddy-viscosity in the case of wall-bounded laminar flows, resulting in an accurate reproduction of the laminar to turbulent transition. The tensor operator $\overline{O P}$ in Eq. 6 for the WALE model reads then:

$$
\overline{O P}_{W}=\frac{\left(\overline{S_{i j}^{d}} \overline{S_{i j}^{d}}\right)^{3 / 2}}{\left(\overline{S_{i j}} \overline{S_{i j}}\right)^{5 / 2}+\left(\overline{S_{i j}} \overline{S_{i j}^{d}}\right)^{5 / 4}}
$$


The model constant has been set to $C_{W}=0.575$ as an average of the values reported in [22]. When the distance from a solid wall approaches zero, the invariant (13) scales as $y^{2}$ so that the WALE operator $\overline{O P}_{W}$ scales as $y^{3}$ reproducing the proper behavior of the eddy-viscosity in near-wall regions.

\section{The Dynamic WALE SGS Model}

The dynamic procedure proposed by Germano [31] to develop the dynamic Smagorinsky SGS model can be applied also to the WALE model. In such case the expression 8 is replaced by:

$$
C_{d W}^{2}=\frac{1}{2} \frac{<L_{i j} M_{i j}^{W}>^{+}}{<M_{i j}^{W} M_{i j}^{W}>}
$$

where $L_{i j}$ is the same as 9 and the superscript + denotes the usual positive-clipping procedure. The operator $M_{i j}^{W}$ then reads:

$$
M_{i j}^{W}=\hat{\Delta}^{2} \widehat{\hat{P P}^{W}} \hat{\overline{S_{i j}}}-\left(\Delta^{2} \widehat{P^{W}} \overline{S_{i j}}\right)
$$

As pointed out by Baya Toda et al. [26] the dynamic version of the WALE model produces too high values of the constant $C_{d W}$ at the walls and a poor prediction of the mean velocity. The reason for this has been found by the Authors [26] who demonstrated that the dynamic procedure tends to alter the proper near-wall $y^{3}$ behavior of the model constant leading to a $y^{0}$ scaling in this region. Since the laminar viscosity is predominant in the proximity of solid boundaries, the Authors [26] concluded that there is no reason to dynamically adapt the model constant there. In order to identify the near-wall region it has been developed a sensor capable of detecting the presence of a wall with no need to have an a-priori knowledge of the geometry that can be arbitrary complex. The sensor has been named "Shear and Vortex Sensor" (SVS) and has the following formulation:

$$
S V S=\frac{\left(\overline{S_{i j}^{d}} \overline{S_{i j}^{d}}\right)^{3 / 2}}{\left(\overline{S_{i j}^{d}}{\overline{S_{i j}^{d}}}^{3}\right)^{3 / 2}+\left(\overline{S_{i j}} \overline{S_{i j}}\right)^{3}}
$$

The SVS invariant is bounded by 0 - 1, where the lower value corresponds to pure shear and the higher corresponds to pure rotating flows. Thanks to this property the computational domain can be ideally split into two regions: the near-wall region, identified by $S V S<0.09$ (as proposed by the Authors [26]), where the model constant is fixed at its standard value, and a bulk region where the model constant is evaluated dynamically. The (modified) dynamic WALE SGS model can be summarized as follows:

- if $S V S<0.09$ :

$$
C_{d W}^{2}=\frac{1}{2} \frac{<L_{i j} M_{i j}^{W}>^{+}}{<M_{i j}^{W} M_{i j}^{W}>}
$$

- if $S V S \geq 0.09$ :

$$
C_{d W}^{2}=0.25
$$

\section{The Sigma SGS Model}

The Sigma SGS model has been recently proposed by Baya Toda et al. [27] as an upgrade of the WALE model.

In [27], the Author highlights some deficiencies of the WALE model: it can be shown that it produces nonzero eddy viscosity in simple flow configuration such as solid rotation and axisymmetric expansion or contraction, where the sub-grid scale activity should ideally be zero. The Authors developed the model in order to force the operator $\overline{O P}$ to have the following properties:

- P1: to behave as $y^{3}$ in proximity of walls.

- P2: to vanish in the case of solid body flow rotation (like the Smagorinsky model) and in the case of pure shear (like the WALE and Vreman [33] models). In general, the operator should vanish in the case of any two-dimensional flow.

- P3: to vanish where the resolved flow field is either in a pure axisymmetric or in a isotropic contraction/expansion configuration. 
In the Sigma model the operator $\overline{O P}$ takes the following form:

$$
\overline{O P}^{\sigma}=\frac{\sigma_{3}\left(\sigma_{1}-\sigma_{2}\right)\left(\sigma_{2}-\sigma_{3}\right)}{\sigma_{1}^{2}}
$$

where $\sigma_{1}, \sigma_{2}$ and $\sigma_{3}$ are the three singular values of the velocity $\overline{g_{i j}}$ gradient tensor. By definition these values are always positive and equal to the square root of the eigenvalues of the matrix $\mathbf{G}=\mathbf{g}^{t} \mathbf{g}$, which are always positive because $\mathbf{G}$ is symmetric semi-definite positive. Analyzing the asymptotic behavior of the singular values at walls, it can be shown that:

$$
\sigma_{1}=O\left(y^{0}\right) ; \sigma_{2}=O\left(y^{1}\right) ; \sigma_{3}=O\left(y^{2}\right)
$$

Thanks to the relations 21 it is possible to verify that in Eq. $20 \sigma_{3}\left(\sigma_{1}-\sigma_{2}\right)\left(\sigma_{2}-\sigma_{3}\right)$ satisfies the above mentioned properties P1, P2 and P3. The term at denominator is an Authors' choice [27] in order to scale $\overline{O P}^{\sigma}$ to a frequency scale. The model constant is set $C_{\sigma}=1.5$ as proposed in [27].

\section{LES QUALITY}

In the last decade the number of works dealing with LES of ICE flows constantly increased due to the potential this modeling approach has to offer within this field. Despite that, most of the scientific publications lack the resolution quality assessment of the simulations with some noticeable exceptions such as [3], [5], [34] and [19]. In the following paragraphs, LES quality assessment parameters are presented and discussed. It must be noted however that these represent a subset of a larger number of resolution tests available. As far as ICE flows are concerned, some of these tests become of limited interest because of the extra computational effort required, such as the Richardson extrapolation methods and, more in general, the two-grid estimators [18].

\section{Fraction of Resolved Kinetic Energy - M}

The amount of turbulent kinetic energy resolved by a simulation is a key aspect for evaluating LES quality. In [17] Pope defines the parameter $M(\bar{x}, t)$ as a measure of the turbulent resolution. Such parameter weights the amount of turbulent kinetic energy of the residual motion with respect to the total turbulent kinetic energy of the flow:

$$
M(\bar{x}, t)=\frac{k_{\text {sgs }}(\bar{x}, t)}{k_{\text {sgs }}(\bar{x}, t)+K_{g s}(\bar{x}, t)}
$$

where $K_{g s}(\bar{x}, t)$ is the resolved turbulent kinetic energy and $k_{s g s}(\bar{x}, t)$ is the residual motion turbulent kinetic energy. The value of $M$ is bounded between 0 and 1 . When $M=1$ a RANS simulation is retrieved, while for $M$ approaching zero, the simulation tends to a DNS. The smaller the parameter $M$ is, the higher the resolution of the turbulent motions is. Pope suggests a threshold value of $M<0.2$ as a reasonable resolution limit for LES.

It must be noted, however, that the parameter $M$ alone is not sufficient to evaluate the accuracy of the simulation with respect to the actual flow since $M$ does not account for the fraction of turbulent kinetic energy lost due to numerical dissipation. Furthermore, a high value of $M$ at a certain point in the domain doesn't necessarily mean that in such location the grid needs refinement because the fraction of turbulent kinetic energy could be affected by the filter size in upstream locations and / or earlier in time.

A wall-bounded flow can be fully resolved only if the near-wall region is discretized with a properly refined mesh in all three directions given the three-dimensional nature of turbulence. In ICE flows such a requirement is hard to fulfill due to the huge computational effort needed. In consideration of this and trying to address feasible modeling strategies, all the turbulence models proposed in the present work make use of wall functions, if not otherwise stated.

\section{Length Scale Resolution - LSR}

The Length Scale Resolution ( $L S R$ ) has been introduced by Brusiani et al. in [20] attempting to estimate the attitude of the grid to properly resolve the scales of fluid motion up to the inertial subrange. It is defined as the ratio between the actual filter size $\Delta$ and (an estimate of) the inertial subrange lower limit $l_{D I}$ :

$$
\operatorname{LSR}(\bar{x}, t)=\frac{\Delta}{l_{D I}(\bar{x}, t)}
$$


where $\Delta=\sqrt[3]{\Delta x \Delta y \Delta z}$ and $l_{D I} \approx 60 \eta$ with $\eta$ being the Kolmogorov microscale length [?]. To be noted that such an estimate of $l_{D I}$ is based on scaling assumptions of isotropic turbulence under equilibrium conditions. In the present work the above parameters are estimated as follows:

$$
\begin{gathered}
\Delta=\sqrt[3]{\Delta x \Delta y \Delta z} \\
\eta(\bar{x}, t)=\nu^{3 / 4} \varepsilon(\bar{x}, t)^{-1 / 4} \\
\varepsilon(\bar{x}, t) \approx \frac{C_{\varepsilon} k(\bar{x}, t)^{3 / 2}}{\Delta}
\end{gathered}
$$

The $L S R$ parameter is therefore defined as the ratio between the actual filter dimension and the characteristic length of the inertial range motions. Such parameter correlates the filter dimension $\Delta$ to the fraction of resolved energy spectrum and is therefore useful to evaluate the optimality of the computational domain discretization, identifying regions that could need refinement. While having $L S R=1$ means complete length scale resolution up to the dissipative range, accordingly to the Authors' findings [20], $L S R<5$ is a reasonable threshold for an adequate energy resolution level at an affordable computational cost.

\section{Viscosity Ratio $-\nu_{S G S} / \nu$}

The ratio between the sub-grid viscosity $\nu_{S G S}$ and the molecular viscosity $\nu$ is representative of the degree of modeling in a LES simulation:

$$
s^{\prime}=\frac{\nu_{S G S}}{\nu}
$$

Several Authors prefer to weight the amount of turbulent viscosity with respect to the effective viscosity defining the following subgrid activity parameter:

$$
s^{\prime \prime}=\frac{\nu_{S G S}}{\nu_{S G S}+\nu}
$$

However, as Celik pointed out in [18], since $\nu_{S G S}>>>\nu$ the $s^{\prime \prime}$ parameter is often pretty close to unity and not really sensitive to grid resolution. For such reason in the present work the $s^{\prime}$ formulation has been used. Values of $s^{\prime}<10$ are considered sufficient to obtain an accurate LES as shown by Durbin in [36].

\section{Two Grid Estimator - $L E S_{I Q k}$}

The $L E S_{I Q k}$ parameter has been proposed by Celik [18] who defined it as the ratio between the resolved turbulent kinetic energy $k_{r e s}$ and the total turbulent kinetic energy $k_{t o t}=k_{r e s}+k_{S G S}+k_{n u m}$ :

$$
L E S_{I Q k}=\frac{k_{r e s}}{k_{t o t}}=\frac{k_{r e s}}{k_{r e s}+k_{S G S}+k_{n u m}}
$$

When $L E S_{I Q k}=0$ a RANS simulation is retrieved, while the LES simulation approaches a DNS when $L E S_{I Q k}$ tends to unity. By assuming that the effective subgrid turbulent kinetic energy $k_{e f f, S G S}=k_{S G S}+k_{n u m}$ scales with the mesh size, the Author reformulates Eq. 29 as:

$$
L E S_{I Q k}=\frac{k_{r e s}}{k_{r e s}+a_{k} h^{P}}
$$

where $a_{k}$ is a coefficient to be determined, $p$ is the formal order of accuracy of the numerical scheme and $h$ is the grid size. To be noted that the formulation of this quality indicator is well suited for implicitly filtered LES, where a modification of $h$ is directly reflected onto the filter size $\Delta$, and, from this, onto both the numerical and SGS behaviors jointly. Denoting with superscript 1 the finer grid and with superscript 2 the coarser one, Eq. 30 can be reformulated as: 


$$
L E S_{I Q k}=\frac{k_{r e s}}{k_{r e s}+\frac{k_{r e s}^{2}+k_{r e s}^{1}}{\alpha^{P}-1}\left(\frac{h}{h_{2}}\right)^{P}}
$$

where $\alpha=\frac{h_{1}}{h_{2}}>1$ is the grid refinement ratio. Equation 31 can be then rearranged for both grid sizes. Here the expression for the finer grid is reported:

$$
L E S_{I Q k}^{\text {fine }}=\frac{1}{1+\left(1-\frac{k_{r e s}^{1}}{k_{r e s}^{2}}\left(\alpha^{P}-1\right)^{-1}\right)}
$$

In this approach the inherent difficulty of evaluating the code numerical dissipation is hidden into the parameter $a_{k}$ which is computed through a Richardson's extrapolation. The extrapolation is performed with results obtained on two grids, similar in structure but with different refinement levels. Due to this last aspect, the $L E S_{I Q k}$ parameter belongs to the class of two-grid estimators. The procedure is certainly computationally expensive and can lead to misleading results in practical applications as found by Di Mare [19], and this explains its scarce adoption, even in academic works. Despite these issues, this estimator has been used in the present work in order to test its capabilities in evaluating the code's numerical dissipation.

\section{NUMERICAL DISSIPATION AND DISPERSION}

It is well known by literature that numerical schemes tend to introduce numerical dissipation and dispersion on the flow field solution ([37], [21]). In explicit LES analyses, like the ones we dealt with, the dissipation of kinetic energy is provided directly as viscous dissipation or through SGS viscous dissipation, while the numerical dissipation of the code should be kept as low as possible (ideally zero). Furthermore the importance of properly resolving the time evolution of the turbulent flow structures leads to the need of minimize numerical dispersion as well. It is therefore interesting to evaluate the attitude of $O$ penFOAM ${ }^{\mathrm{R}}$ towards both kinds of the aforementioned numerical errors in order to estimate its suitability for LES computations.

The adopted test case consists of a vortex convected by a uniform inviscid flow in a 2D domain as proposed by Yee et al.[38]. Supposing that there are no numerical dissipation or dispersion errors, after one or more convective times $T_{0}$, the vortex profile remain the same as the initial (analytic) one. Similar tests employing a Taylor-Green vortex have been performed by other Authors ([39], [40] and [41]) as a method to assess code dissipation. To be noted that in the following study the dispersion error has been also evaluated as done by Brusiani et al. [20].

The computational domain, reported in Fig. 1, has been discretized using a 2.5D uniform hexahedral mesh with one cell only along z direction, accordingly to OpenFOAM ${ }^{\circledR}$ requirements for pseudo-2D analyses. Three different grid densities have been used employing 12, 24 and 48 points along the vortex radial profile, with mesh sizes of $48 \mathrm{x} 48$, $96 \times 96$ and $196 \times 196$ cells respectively. Given $D_{0}=2 \pi m$ meters, the initial diameter of the vortex, the domain is a square with side of $2 D_{0}$. The domain extent has been chosen in order to minimize the effects of the boundary conditions on the solution and to allow the motion of the vortical structure for a reasonable amount of convective times $\left(T_{0}\right)$.

The initial conditions for the fluid-dynamic vortex flow field were:

$$
\begin{gathered}
u=-\frac{\beta}{2 \pi} y e^{\frac{1-x^{2}-y^{2}}{2}} \\
v=\frac{\beta}{2 \pi} x e^{\frac{1-x^{2}-y^{2}}{2}} \\
p=p_{0}-\frac{\beta^{2}}{8 \pi^{2}} e^{\left(1-x^{2}-y^{2}\right)}
\end{gathered}
$$

where $\beta=5 s^{-1}$ is the vortex strength and $p_{0}=101325 \mathrm{~Pa}$. A constant convective velocity $U_{0}$ of $10 \mathrm{~m} / \mathrm{s}$ has been added along the $x$ direction of the profiles in order to reproduce the convective transport process. Symmetries have been used for the top and bottom domain boundaries while periodic conditions have been applied on the other sides, as shown in Fig. figfig:taylorDomain. 


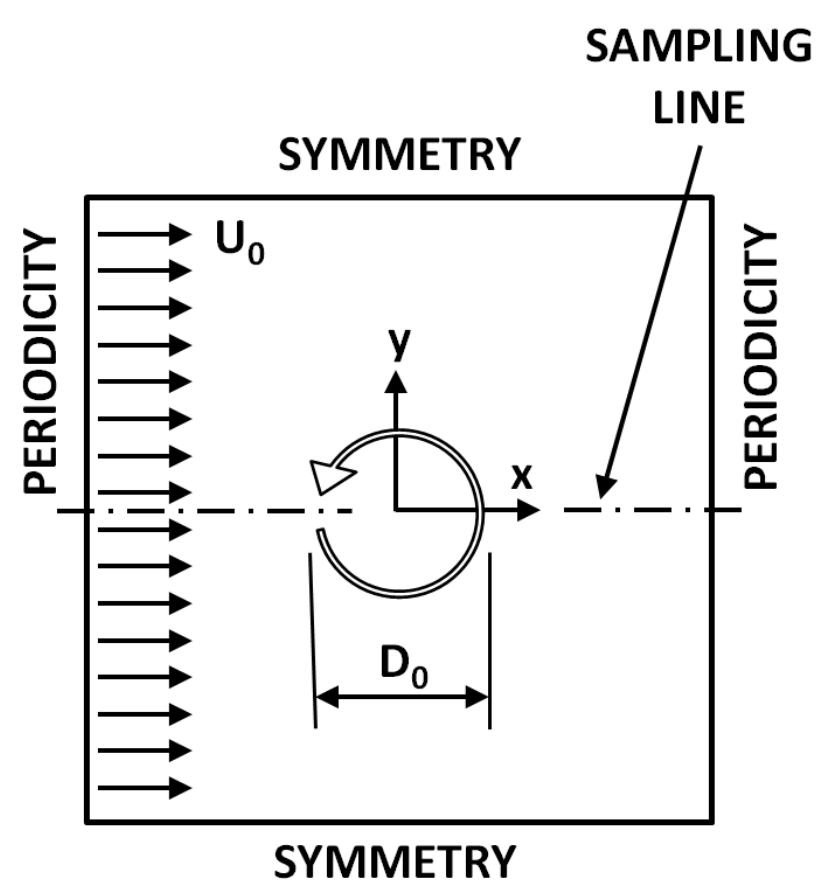

Figure 1: Taylor vortex test setup. Refer to text for details.

The OpenFOAM ${ }^{\circledR}$ solver used is potentialTransientFoam, a solver which was specifically implemented during the present work for the solution of transient inviscid fluid flows. It is based on a second order backward implicit integration scheme for time advancement and employs a Bounded Central Difference scheme (BCS) for pressure and momentum equations. The PISO algorithm provides the pressure- velocity coupling. The same numerical schemes were employed in the 3D LES analyses with viscous flow solvers presented in the following section.

Numerical results are reported in Figures 2 - 7. Figures 2 and 3 show the influence of the grid density on the calculation accuracy by comparing the analytic solution of the vortex transport against the numerical solution after respectively 1 and 5 convective times $T_{0}$. All the three cases have been run with a Courant Number $C_{0}=0.5$ and the pressure profiles are taken from a sampling line coincident with the $x$ axis. It is evident how decreasing the mesh resolution adversely affects the vortex profile: the pressure peak is progressively reduced and the shift in phase with the exact solution increases. Furthermore spurious oscillations of the pressure and velocity field (here not reported) appear when the coarsest mesh is used. In such case the vortex is quickly smeared out and some drift of the vortex core from the centerline tend to occur.

In Fig. 4 and Fig. 5 is shown the influence of the $C o$ on the solution accuracy for the medium density grid. The figures report the vortex pressure profile taken on the same post-processing topology as done for the mesh influence evaluation. The effects of the time discretization are particularly manifest in Fig. 5 where both vortex smearing and shift in phase appear with increasing $C o$.

Finally, Fig. 6 and Fig. 7 summarize the results obtained in terms of numerical dissipation and dispersion. In the former plot the decrease of the vortex total kinetic energy is reported and the effect of the grid resolution on the energy conservation is clearly shown. Moreover the plot suggests that the influence of the space discretization on the vortex kinetic energy preservation is greater than the influence of the time discretization. Such a result is confirmed also by [40] in which the Authors found a significant improvement in the numerical accuracy with the grid resolution. The dispersion error is evaluated by considering the pressure peak vortex drift with respect to the analytic solution, normalized by the vortex original diameter $D_{0}$. In this case both space and time discretization seem to affect the numerical accuracy with a greater influence of the former parameter.

The outcomes of this test give some guidelines on the numerical setup of LES analyses with $O p e n F O A M{ }^{\circledR}$, suggesting to pay attention to the levels of grid refinement and possibly running the simulation with $C o<0.5$. All the tests presented in this work have been carried out with a maximum $C o$ between 0.3 and 0.5 with some exceptions for the finer grids in which the threshold has been raised to 0.65 to reduce the computing time. Providing specific recommendations for space discretization is not trivial, since it strongly depends on the dimension of the smaller vortical structures that one needs/wants to resolve. 

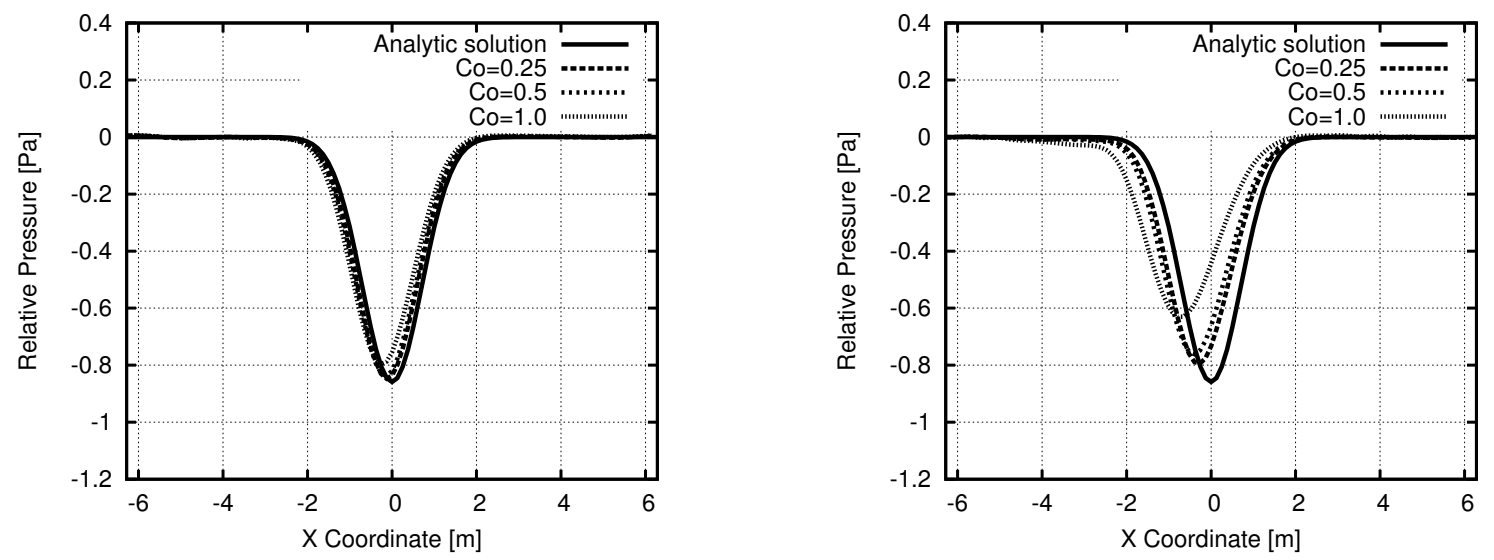

Figure 4: Courant number influence - Relative pressure Figure 5: Courant number influence - Relative pressure profile after one convective time profile after five convective times
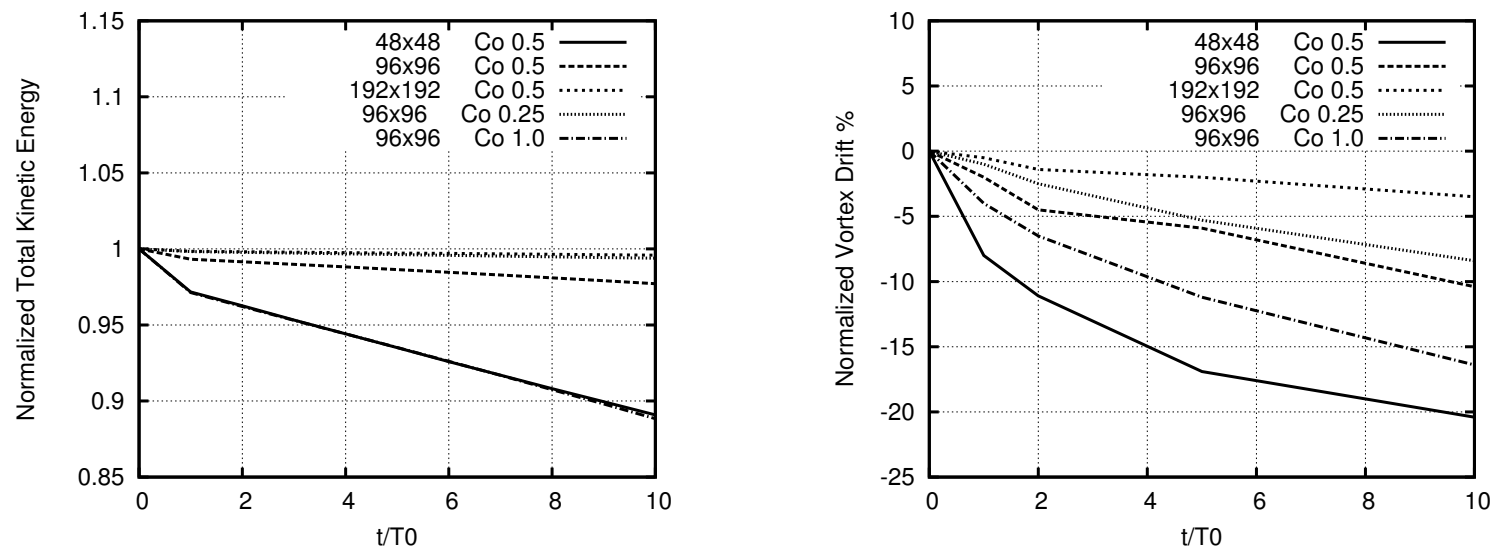

Figure 6: Numerical dissipation as a function of the con- Figure 7: Numerical dispersion as a function of the convecvective time tive time
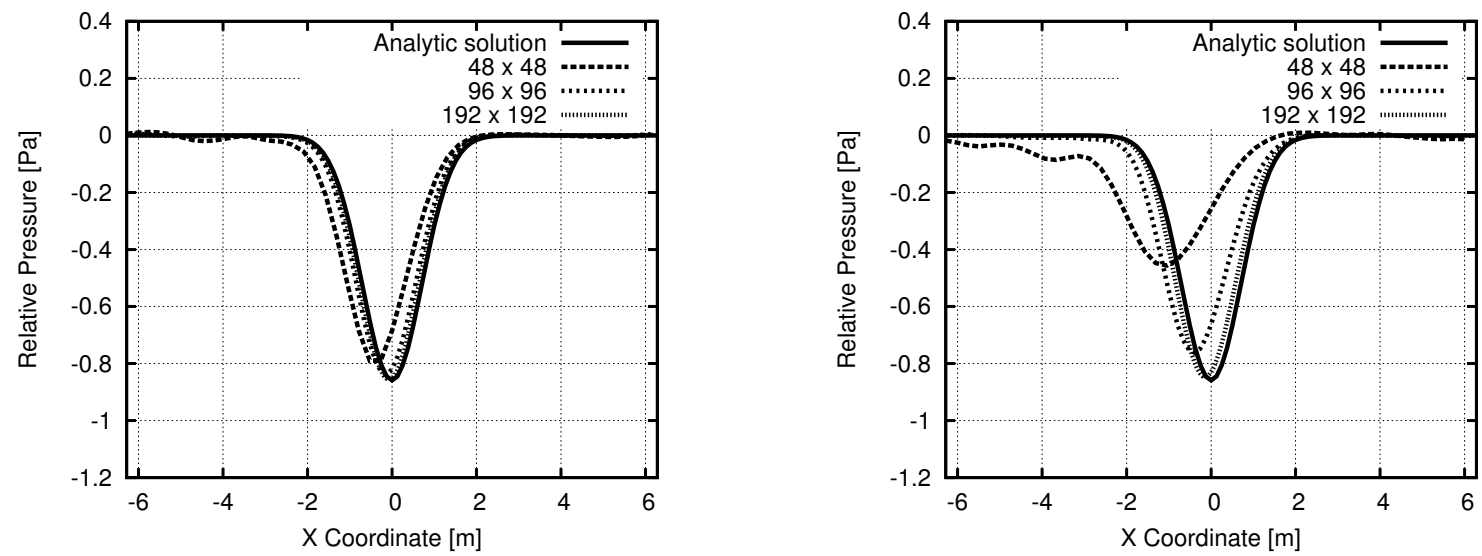

Figure 2: Mesh influence - Relative pressure profile after Figure 3: Mesh influence - Relative pressure profile after one convective time five convective times

Moreover, in case of ICE simulations the characteristic length scale varies with operation type (motored/fired), engine regime and engine phase (see [42], [43], [44], [45] or [46] for more details on this issue). Once the characteristic di- 
mensions of the structures intended to be resolved are known, the aforementioned findings could provide an indication of the amount of points needed for proper resolution, and, from this, an estimate of the grid spacing.

\section{DELLENBACK ABRUPT EXPANSION}

\section{Test Case and Computational Setup}

The implemented SGS models have been firstly validated on a test case related to a swirling flow through a sudden expansion that has been experimentally investigated by Dellanback et al. [30]. The swirl number, defined as:

$$
S=\frac{\int_{0}^{R} U_{\theta} U_{z} r^{2} \mathrm{~d} r}{R \int_{0}^{R} U_{z}^{2} r \mathrm{~d} r}
$$

was approximately 0.98 , based on the inlet radius $R=D_{0} / 2 . U_{\theta}$ and $U_{z}$ are respectively the time-averaged tangential and axial velocity components. The Reynolds number, based on the inlet diameter $D_{0}$ and the inlet bulk velocity was $R e=30000$. Even if the case may seem a little far from being a flow of interest for engine application, it has several features that can be found in real engine flows, such as swirl, flow detachment and recirculating regions. In fact, the case has already been subject of numerical investigation by other Authors, amongst which Schlueter [47], Javadi [48] and Piscaglia [49].

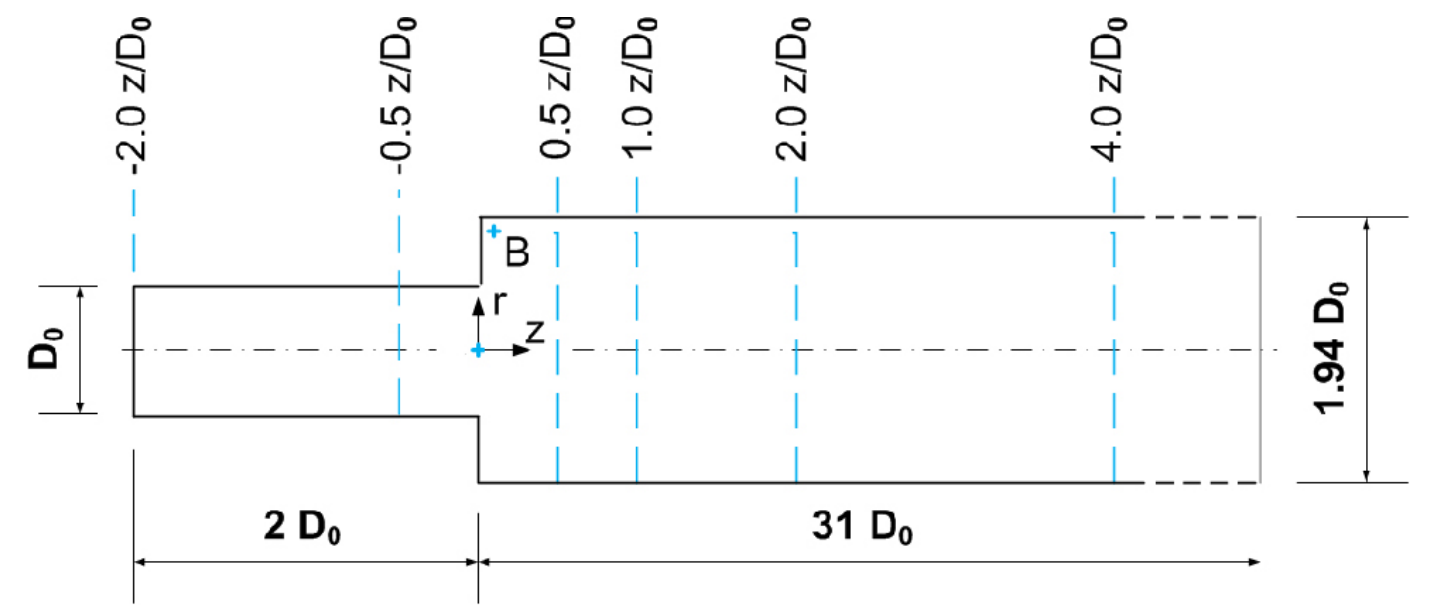

Figure 8: Geometry of the Dellenback test case and probe locations

The geometry of the case is reported in Fig. 8. The inlet duct has a diameter $D_{0}=0.0508 \mathrm{~m}$ and a length of $31 D_{0}$ with the expansion located $15 D_{0}$ downstream the swirl generator. The duct diameter after the expansion is $D_{1}=0.0985 \mathrm{~m}$, giving an expansion ratio of 1:1.94. The experiments have been performed using water and the swirl has been generated supplying a portion of the flow through tangential slots. Axial and tangential velocity components of mean and rms components have been measured using Laser Doppler Anemometry (LDA) on different radial stations located through the duct midsection as shown in Fig. 8.

The finite-volume open-source CFD code $O p e n F O A M^{\circledR}$ v. 2.3.0 has been used for the simulations. The temporal derivatives have been discretized using a second-order backward differencing scheme. For momentum convection and diffusion terms a second-order central difference scheme has been employed. The pressure-velocity coupling was performed using the PISO algorithm.

Different types of grids have been used for the simulations:

- Fully hexahedral coarse grid - $0.7 \mathrm{M}$ cells

- Fully hexahedral medium grid - $1.5 \mathrm{M}$ cells

- Fully hexahedral fine grid - 6.0 M cells

- Fully tetrahedral medium grid - $1.5 \mathrm{M}$ cells 
The hexahedral grids are all block-structured with a slightly higher resolution near the expansion. No near-wall mesh refinement has been explicitly applied and wall functions have been used to handle such region. This approach is justified by the needs of ICE flow computations where a full near-wall resolution is extremely difficult to achieve, at least in real-world cases. The $y^{+}$in the expansion region walls ranges from 15 to 50 depending on the grid.

Time discretization has been performed ensuring a maximum $C o<0.5$ in light of the considerations made in the previous section, employing timesteps in the range of $10 e-4-10 e-5 s$.

Both the solver and the turbulence models adopt an incompressible formulation. Even if compressibility effects in ICE flows are important, the objective of this early stage work was the evaluation of the two implemented SGS models (dynamic Wale and Sigma), whose performance does not depend on the compressibility of the flow.

At the pipe inlet mean velocities profiles based on experimental data have been mapped onto the domain. No turbulent fluctuations have been explicitly set. The choice of using a "pseudo-laminar" inlet boundary condition was motivated by the high level of swirl, capable of inducing a fast transition to turbulence, as shown in [47]. At the expansion outlet a homogeneous zero-gradient boundary condition has been set for velocity and a fixed value of zero relative pressure has been set. Finally, a no-slip condition has been applied at walls.

\section{Results - Velocity Profiles}

A first evaluation of the analysis results is made by comparing the velocity mean and RMS profiles at different locations with the corresponding experimental data. The profiles have been averaged over 12 mean flow advection times and circumferentially averaged. Figure 9 shows the velocity profiles and the corresponding turbulent fluctuation levels obtained with the different SGS models on the coarse grid. Looking at the profiles at $\mathrm{z} / \mathrm{D}=-0.5$, it can be noticed that even if employing a pseudo-laminar inlet condition the mean profiles are correctly predicted and the turbulent fluctuations have grown pretty close to the experimental ones thanks to the high swirl level, as explained in the previous section. The evaluation of the profiles downstream the expansion reveals an overall good agreement with the measurements with some deficiencies in both the mean and RMS axial profiles close to the pipe axis. The Wale and the Sigma models appear to perform slightly better than the other models. The dynamic Smagorinsky seems to be the less accurate model, in particular close to the walls. This is not surprising since it is known by literature to over-predict viscosity in these regions. 

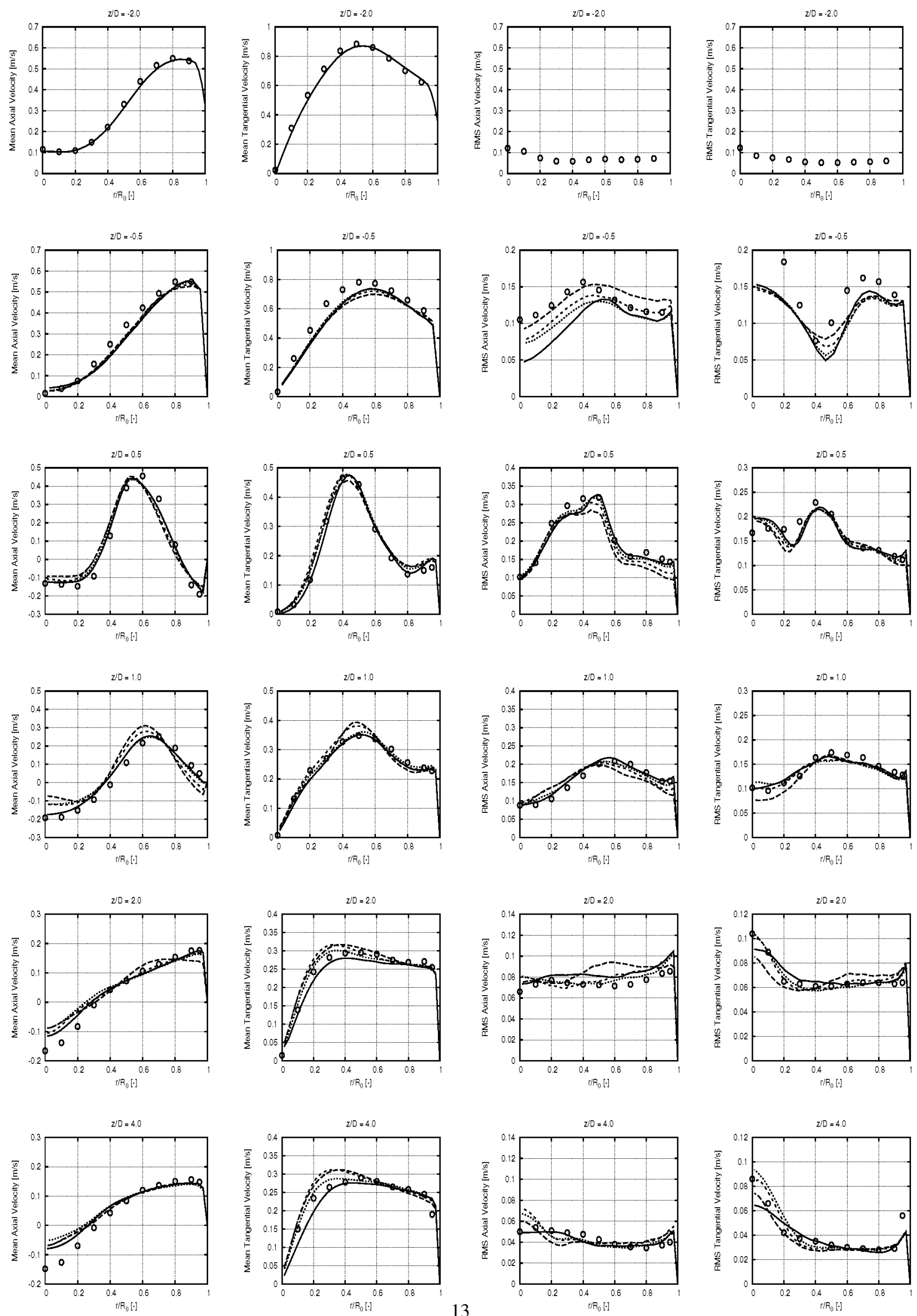

Figure 9: Mean and rms velocity profiles at different locations for the coarse mesh. Legend: open circle: experiment, large dash: dynamic Smagorinsky, small dash: dynamic Wale, continuous: Wale, dots: Sigma 
Figure 10 displays the velocity profiles obtained with the Sigma SGS models for the three hexahedral grids. Both the coarse and the medium density grids seem to resolve with reasonable accuracy the average profiles and RMS fluctuations with a lower agreement at the pipe axis. By contrast the predictions made with the fine grid denote poor agreement with the experimental profiles. This is particularly evident for the RMS fluctuations in the pipe bulk region. The other SGS model profiles (here not reported for the sake of brevity) exhibit a very similar behavior. A possible reason for this could be an improper tuning of the models constants $\left(C_{d S}, C_{W}, C_{d W}\right.$ and $\left.C_{\sigma}\right)$. In particular, too low values of such constants could result in an overestimation of the turbulent fluctuations with increasing mesh density, as explained by Piscaglia et al. in [5] facing a similar issue. This aspect is still under investigation in order to better understand the cause of the problem and a possible solution will be proposed in future works.
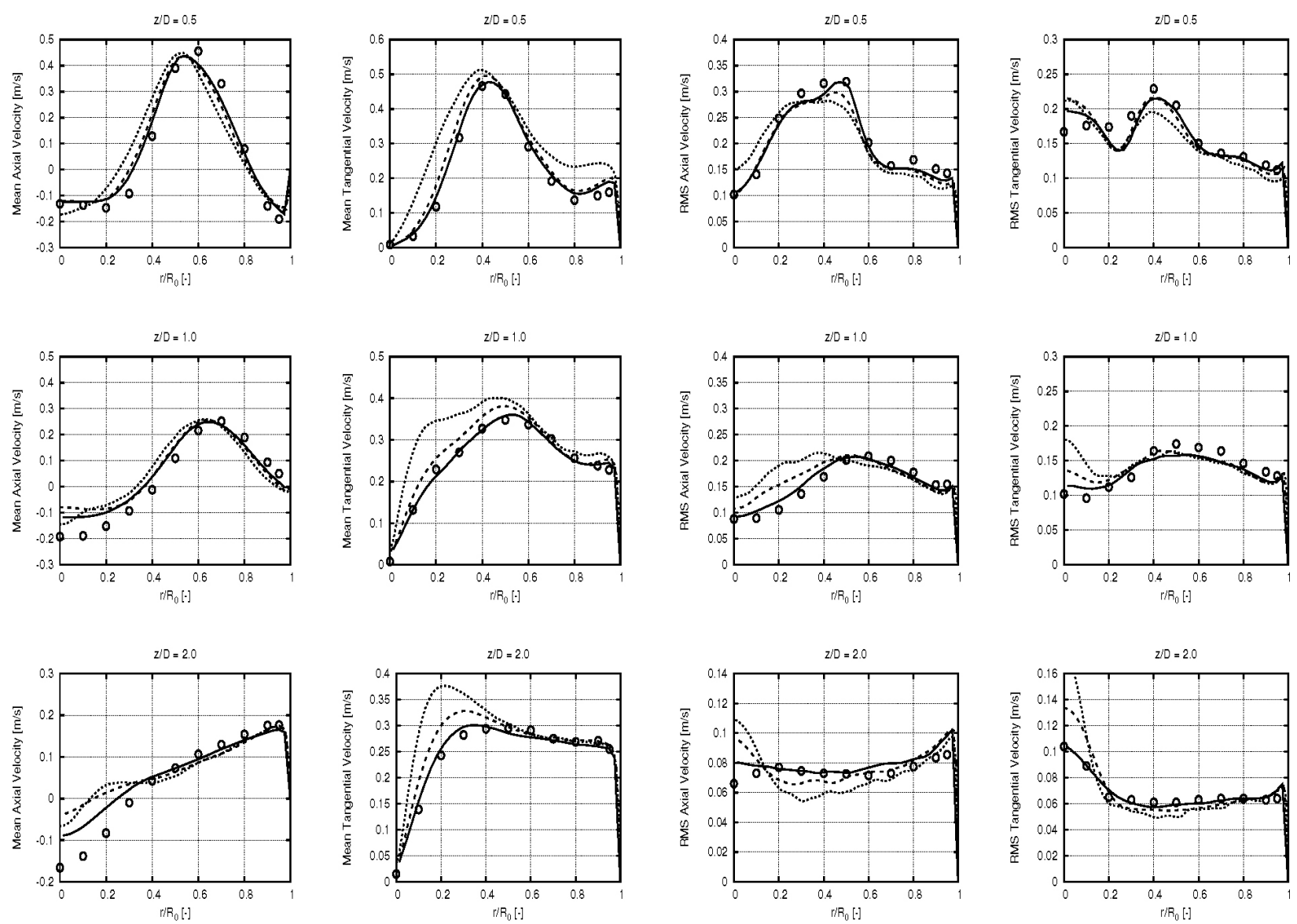

Figure 10: Mean and rms velocity profiles for different grid densities (Sigma model). Legend: open circle: experiment, continuous: coarse grid, small dash dynamic: medium grid, dots: fine grid

The effect of employing grids with different types of element is evaluated through Figure 11 which displays the velocity profiles obtained with the Sigma SGS model on the medium density hexahedral grid and on a uniform tetrahedral grid. The grids have approximately the same number of elements. Both the mean and the RMS profiles obtained on the tetrahedral grid are in good agreement with the experimental data with an accuracy comparable to the hexahedral mesh. This is not surprising since the flow past the expansion, where the sampling stations are located, is generally not instantaneously aligned with the grid elements, being them hexahedra or tetrahedra and this is cause of numerical diffusion adversely affecting the solution accuracy as reported by Brusiani in [3]. 

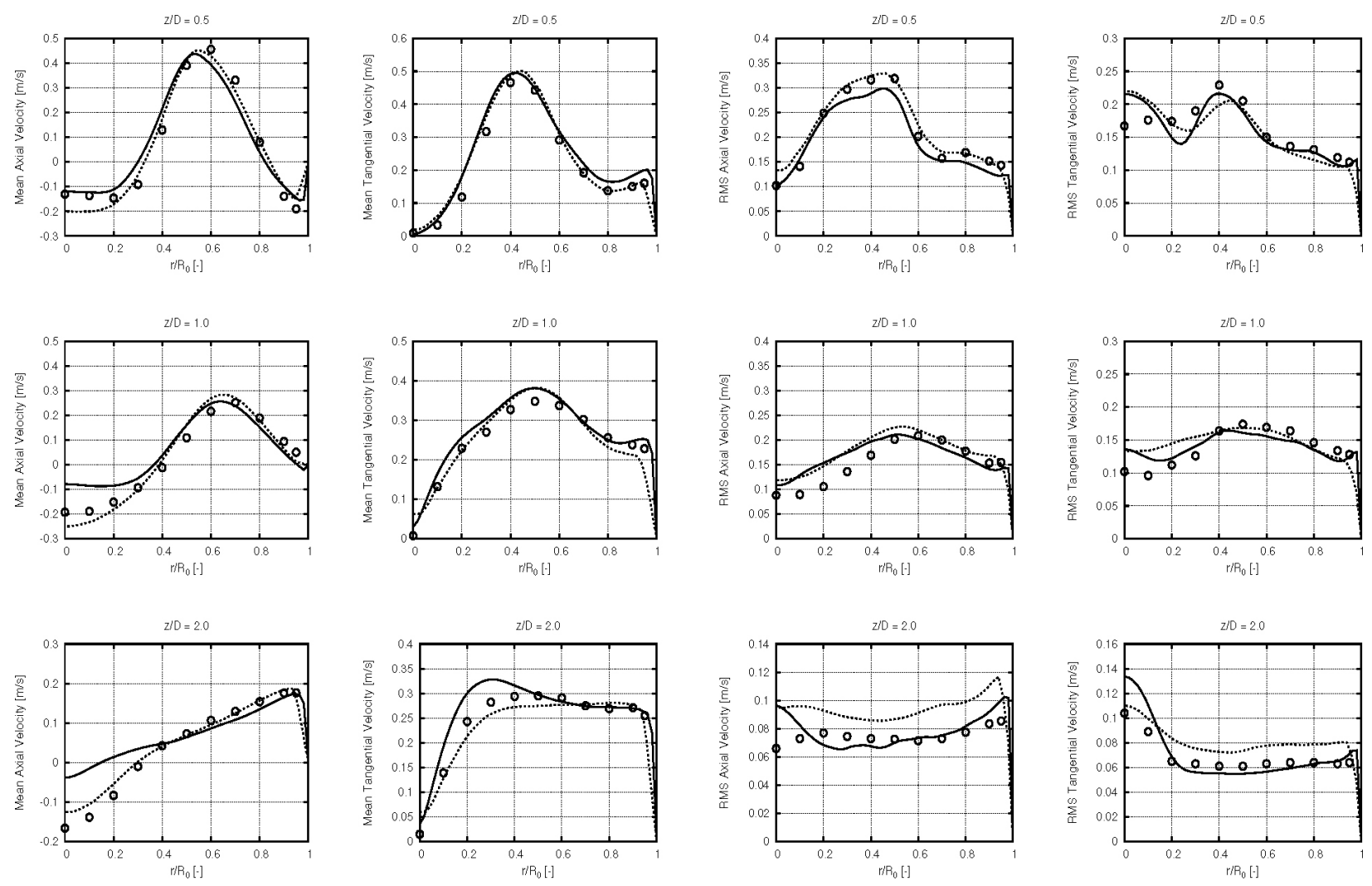

Figure 11: Mean and rms velocity profiles for different grid element type (Sigma model - medium density grid). Legend: open circle: experiment, continuous: hexa, dots: tetra

\section{Results - Quality Assessment}

The quality of LES analyses has been assessed through the parameters proposed in section "LES Quality".

Figure 12 presents the M parameter comparison for the three hexahedral grids. It appears clearly how a refinement of the grid leads to an increase of the fraction of resolved turbulent kinetic energy. The resolution lowers downstream the expansion due to the slight coarsening of grid and close to walls. In this latter region the M parameter is always higher than the threshold due to the insufficient resolution obtained by using a wall modeling approach and this was somehow expected. 

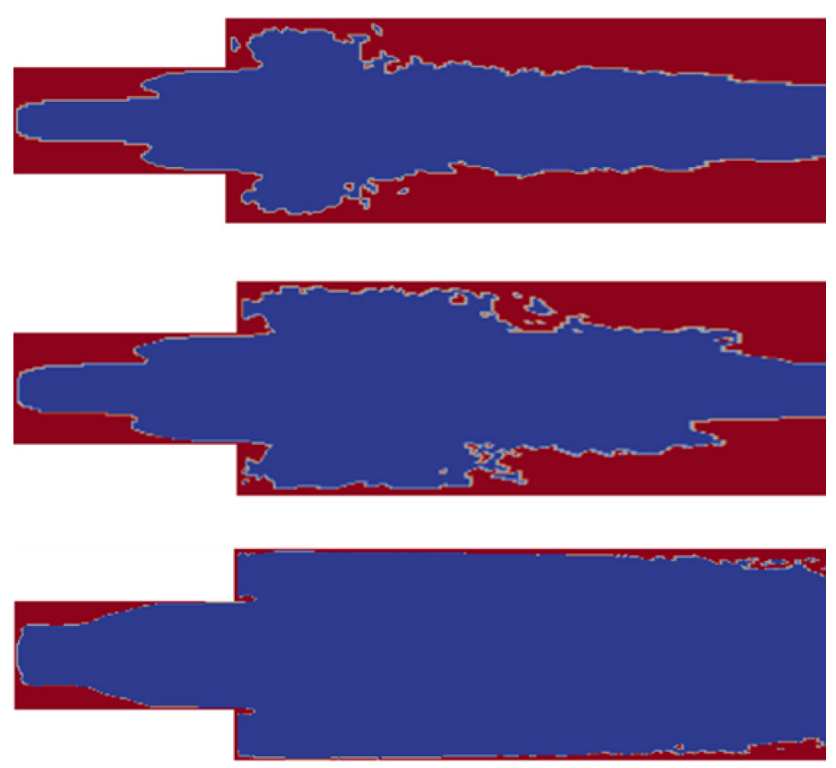

Figure 12: M parameter for different grid densities (Sigma SGS model): top - coarse grid, middle - medium grid, bottom - fine grid. In red the regions where $M>20 \%$

Figure 13 shows the LSR parameter contour for the aforementioned grids. The scale resolution improves with grid refinement and the LSR values for the bulk regions are always smaller than 3 with some exception for the coarse grid. Conversely near wall region suffer a lack of resolution for the near wall modeling just mentioned above.
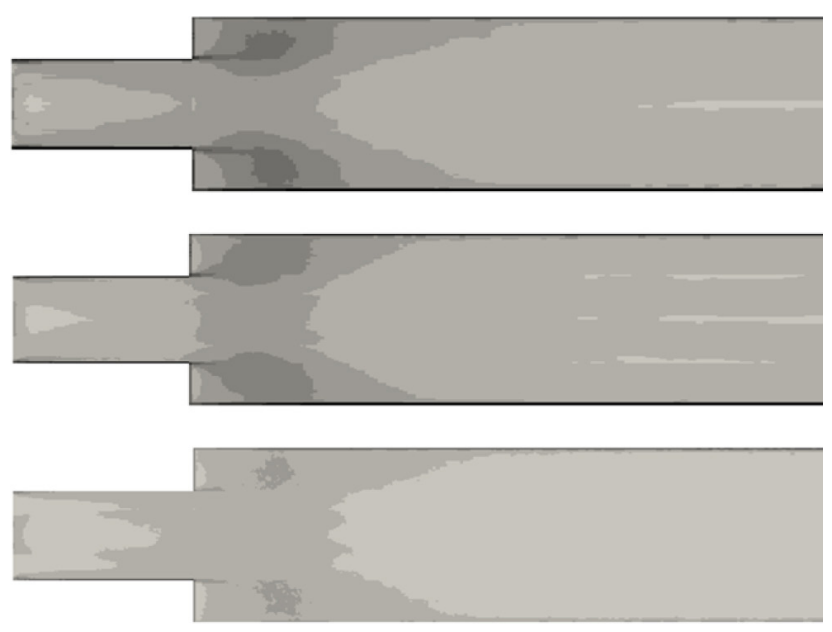

\section{LSR}

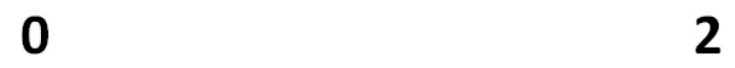

Figure 13: LSR parameter for different grid densities (Sigma SGS model): top - coarse grid, middle - medium grid, bottom - fine grid.

Viscosity ratio contours are proposed in Figure 14 where the contribution of the subgrid scales visibly lowers with grid refinement. High levels of turbulent viscosity tend to remain right behind the expansion close to walls. 

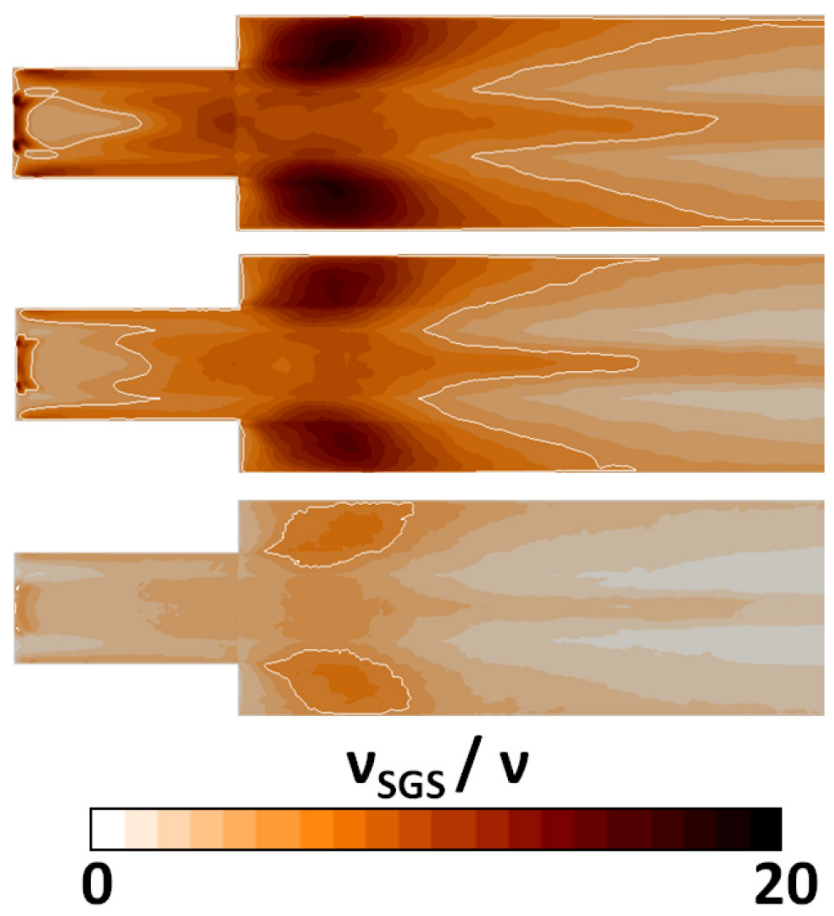

Figure 14: $n u_{S G S} / \nu$ parameter for different grid densities (Sigma SGS model): top - coarse grid, middle - medium grid, bottom - fine grid. The white contour highlights the isosurface $n u_{S G S} / \nu=5$.

Figure 15 presents the trends of the two-grid quality estimator $L E S_{I Q k}$ for three different sampling stations past the expansion. The index appears to drop in almost all cases at the pipe axis and close to wall, probably due to insufficient resolution.

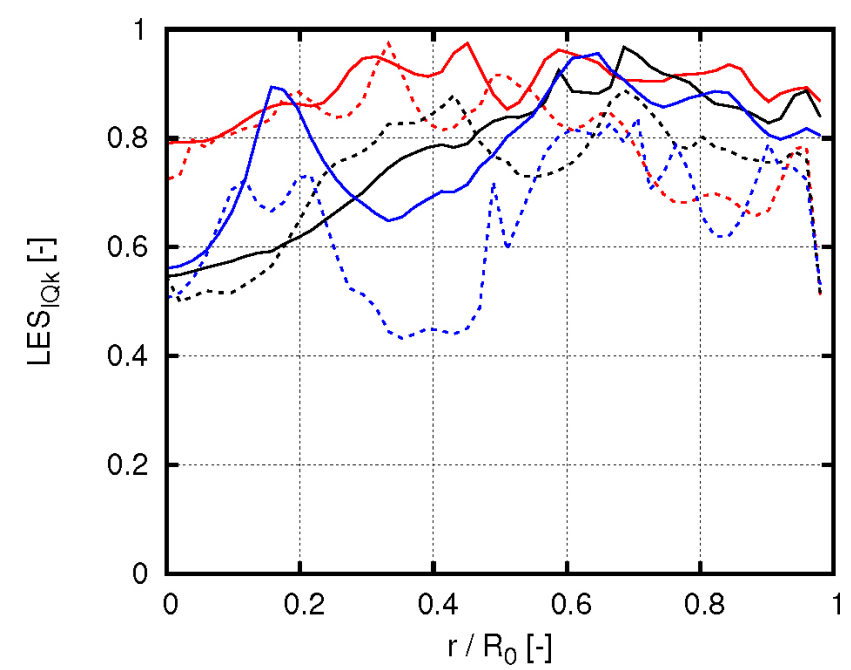

Figure 15: $L E S_{I Q k}$ parameter at different locations (Sigma SGS model): red - z/D = 0.5, black - z/D = 1.0, blue - z/D $=2.0$. Dashed line - medium mesh, Solid line - fine mesh.

Furthermore $L E S_{I Q k}$ levels increase with grid refinement even if not in a monotonic way. Such issue has already been tackled by Di Mare, who in [19] explains how an insufficient statistical sample can lead locally to $k_{\text {res }}^{1}<k_{r e s}^{2}$.

Finally, comparison maps between the medium and the fine grid $L E S_{I Q k}$ are presented in Figure 16 . The parameter distributions, in particular for the medium grid, seem to be quite noisy, maybe due to the aforementioned insufficient 
statistical sampling. Despite being not so easy to read, both maps present lower resolution levels close to the walls and at the pipe axis, following the trends of the other estimators.

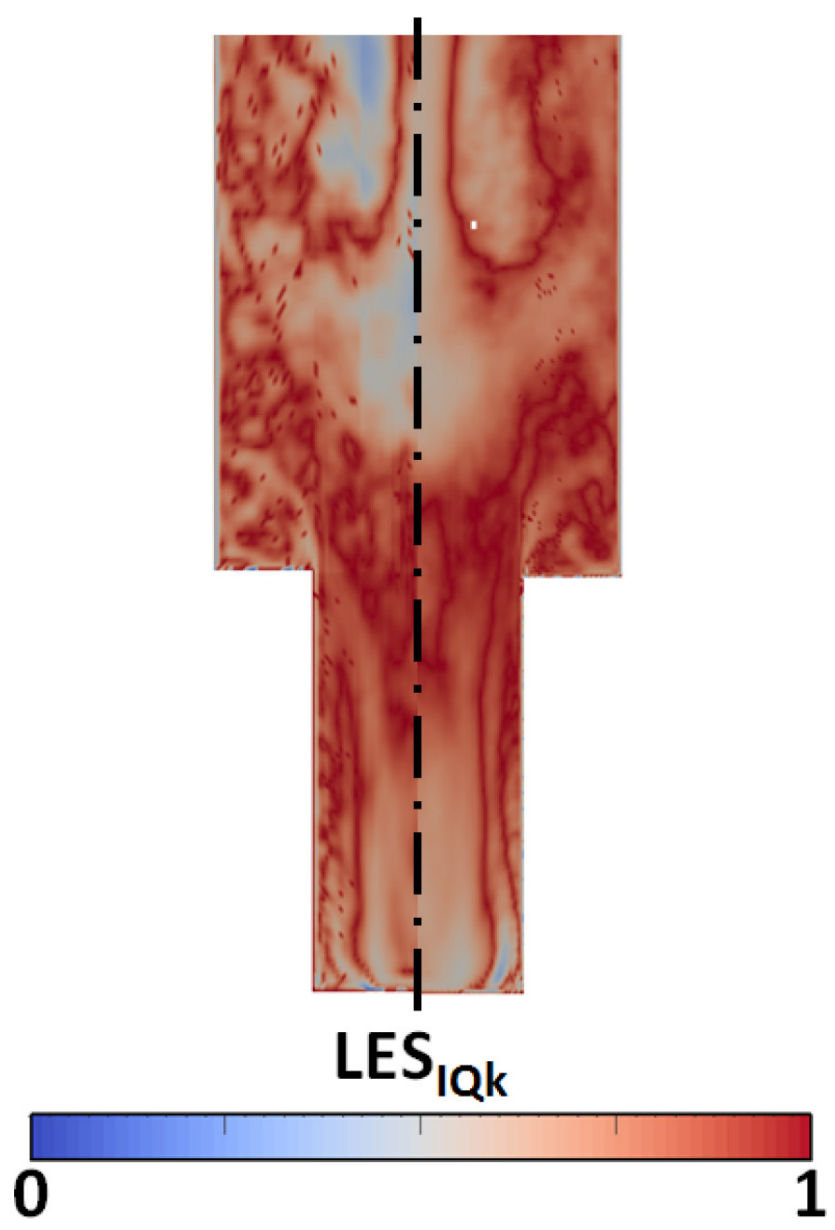

Figure 16: $L E S_{I Q k}$ parameter comparison maps (Sigma SGS model): medium grid (left) and fine grid (right).

The trends of the quality estimators here presented indicate that the finer the grid is, the greater the level of resolution provided is. Despite this, the overall quality of the analyses, evaluated in terms of agreement between the simulations and the experiments, tends to diminish when increasing the grid density. Other aspects, such as the grid quality and the SGS models constants, could affect such agreement. Furthermore, all the estimators returned qualitatively comparable results with differences lying in the estimator range of values and sensitivity. This leads to conclude that these LES quality estimators cannot be used as tools for validation of simulations, even if they are all capable of characterizing the flow field in terms of grid resolution adequacy. They can be therefore usefully employed for comparing grids obtained with the same discretization procedure and/ or to identify potential regions that need to be refined in order to improve the resolution.

\section{Results - Energy Spectra}

Simulations energy spectra have been evaluated at point B of the domain (see Figure 8) which is located close to the walls, right past the expansion. Figure 17 shows the effect of the grid density on the resolved kinetic energy spectrum. All the three hexahedral discretization seems to exhibit a $-5 / 3$ power law inertial subrange behavior but the denser is the grid the better the asymptotic trend is followed, as expected. The influence of the element shape on the spectra is analyzed in Figure 18 in which it appears evident a faster depauperation of the spectrum energy content when the tetrahedral grid is employed. Finally, Figure 19 demonstrate a substantial lack of influence of the SGS model on the spectrum shape. 


\section{Results - Pressure SPD}

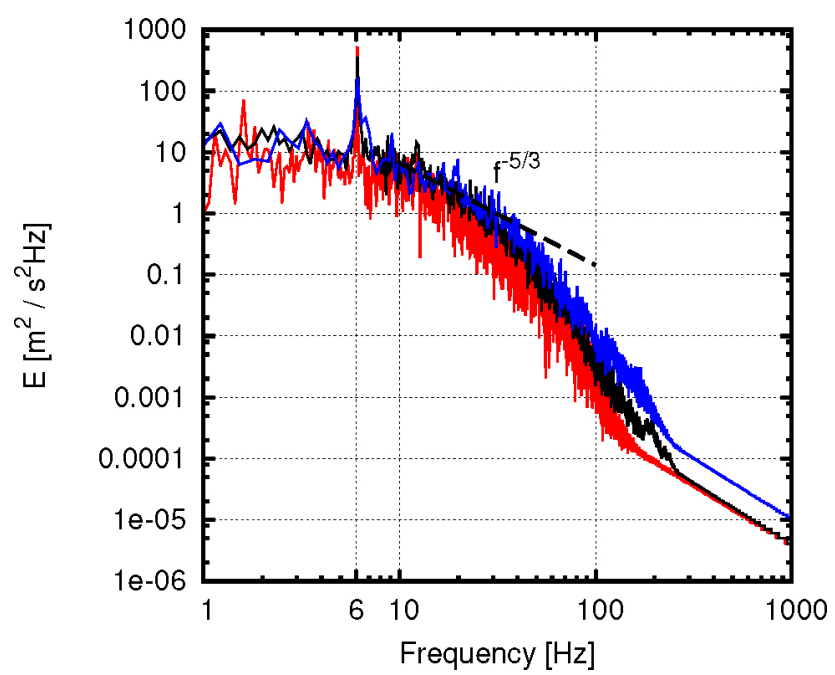

Figure 17: Turbulent kinetic energy spectra for different grid densities (Sigma SGS model): coarse grid (red), medium grid (black), fine grid (blue).

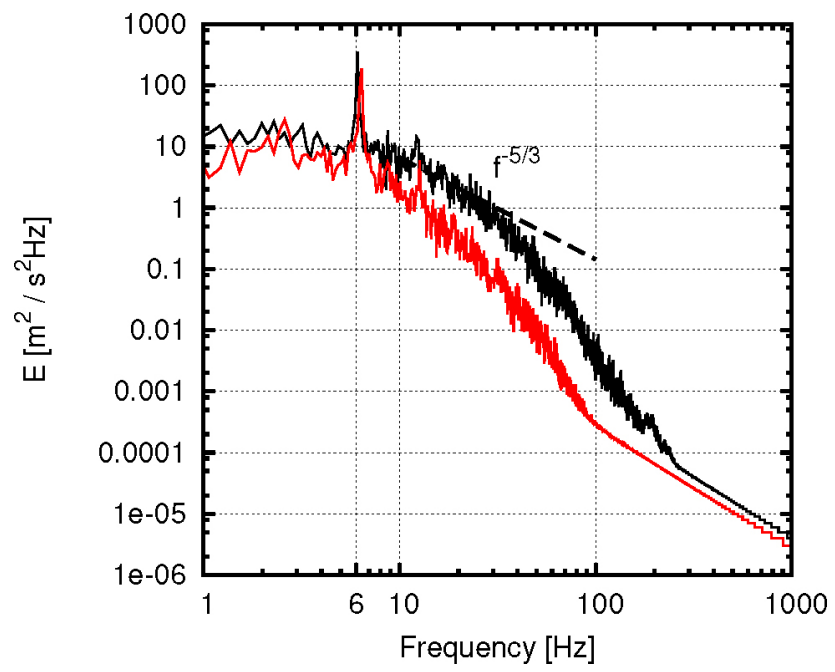

Figure 18: Turbulent kinetic energy spectra for different grid element type (Sigma SGS model): tetrahedral grid (red), hexahedral grid (black). 


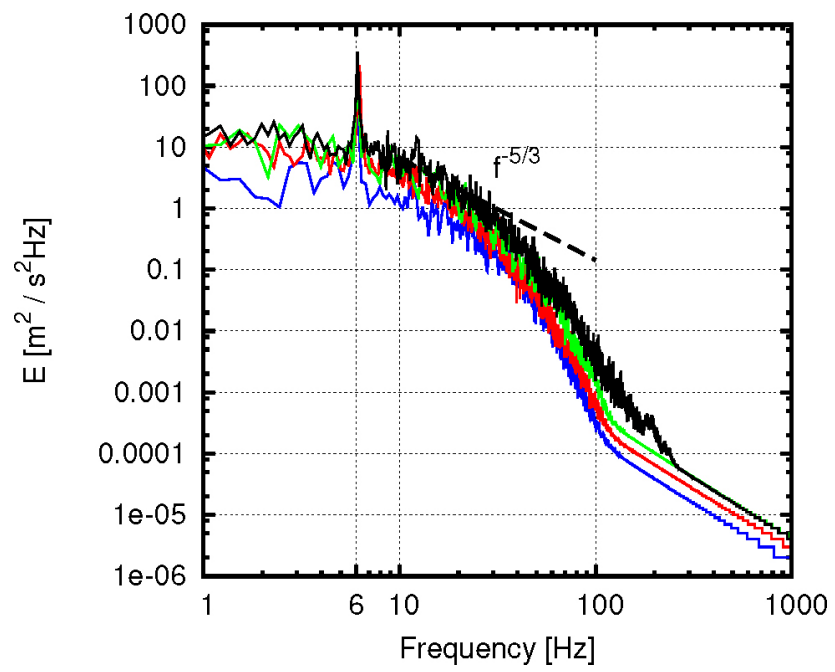

Figure 19: Turbulent kinetic energy spectra for different SGS models: dynamic Smagorinsky (blue), Wale (red), dynamic Wale (green), Sigma (black).

The spectral density of the pressure fluctuations probed at point B are reported in Figures 20 and 21. The plots demonstrate how a reduction in the grid density or the employ of tetrahedral elements tends to reduce the spectral content resolution at higher frequencies. Nevertheless all the discretization show a peak in the spectral content at a frequency of nearly $6 \mathrm{~Hz}$. This frequency is related to the revolution frequency of the helicoidal vortex core as discussed in [50]. Furthermore, the corresponding non-dimensional frequency is at a Strouhal number of $S t=f$. $U_{b} / D=0.985$ in pretty good agreement with the experimental value. Even if less pronounced, the same peak is visible in the energy spectra presented in Figures 17, 18 and 19.

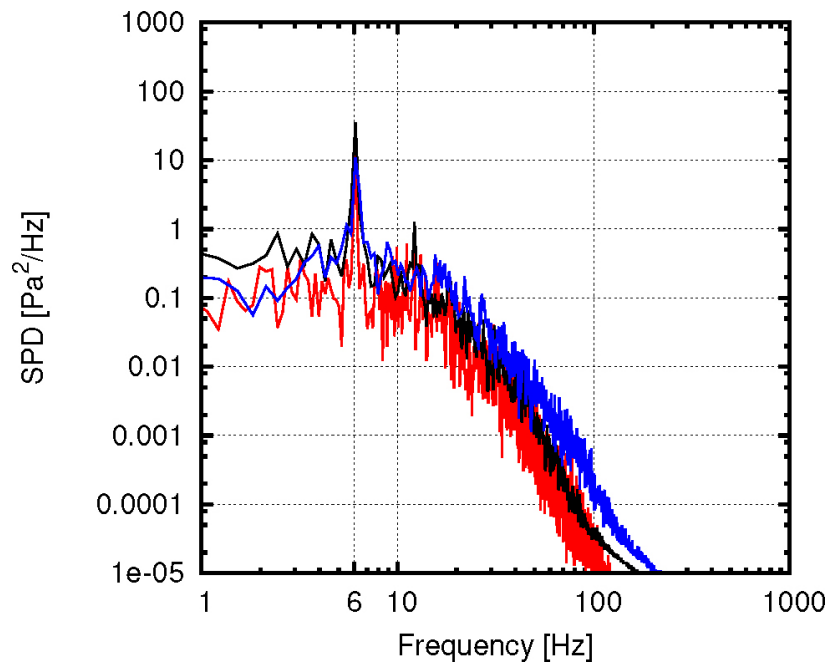

Figure 20: Spectral power density of pressure fluctuations at point B (Sigma SGS model): coarse grid (red), medium grid (black), fine grid (blue). 


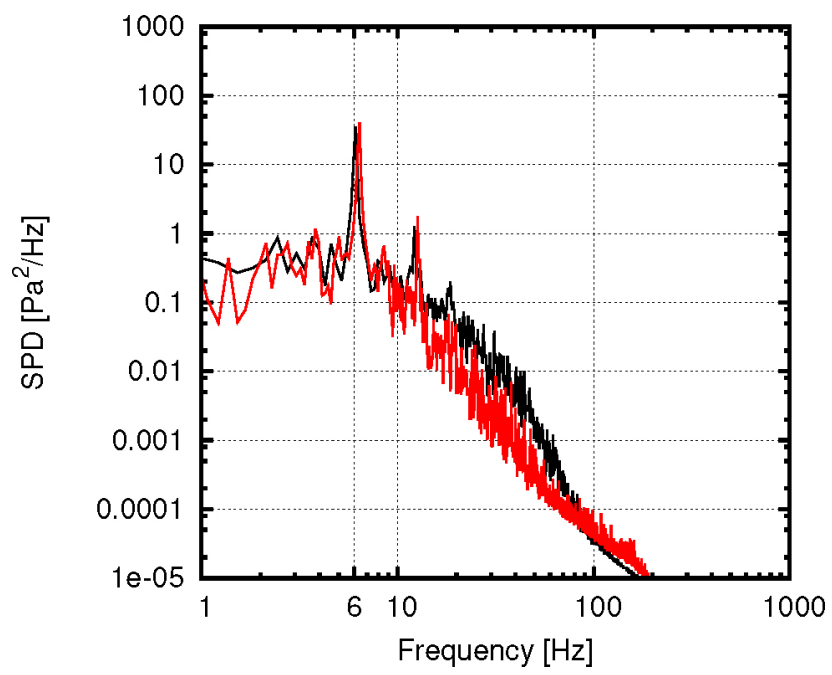

Figure 21: Spectral power density of pressure fluctuations at point B for different grid element type (Sigma SGS model): tetrahedral grid (red), hexahedral grid (black).

\section{COMPUTATIONAL ASPECTS}

The analyses of the present work have been run on the following hardware:

- 2 x Intel Xeon E5-2609 @ 2.40GHz workstation.

- 4 x AMD Opteron 6212 @ 2.60GHz blade.

OpenFOAM ${ }^{\circledR}$ v 2.3.0 was compiled with the GCC v. 4.8.1 linux compiler suite.

A rough estimate of the relative computational cost of the different SGS models is reported in Table 1. The tests have been performed on the Intel workstation employing the medium density grid for the Dellenback case with a serial run. The differential in computational cost of employing the various SGS models has been evaluated counting the number of iterations (time-steps) performed for the same fixed wall clock time. Such estimate is reported in the table as "Computational Intensity". While running the tests, the memory requirement of each run has been tracked. Both the Computational Intensity and the memory footprint (here "Memory Requirement") have been normalized accordingly to the dynamic Smagorinsky SGS model performance. The Wale model seems to be the less demanding SGS model, while the Sigma appears to be the heaviest one. For this latter model, it is in the authors' opinion that the computation of the velocity derivatives tensor eigenvalues is the main cause for the high computational demand. It is however important to note that these tests have been carried out without claiming to be rigorous assessments but just to provide a rough idea of how much does it cost to use a certain model respect to another.

Table 1: Computational requirement of the different SGS models

\begin{tabular}{|c|cccc|}
\hline $\begin{array}{c}\text { Sub-Grid } \\
\text { Scale Model }\end{array}$ & $\begin{array}{c}\text { Dynamic } \\
\text { Smagorinsky }\end{array}$ & Wale & $\begin{array}{c}\text { Dynamic } \\
\text { Wale }\end{array}$ & Sigma \\
\hline $\begin{array}{c}\text { Memory } \\
\text { Requirement }\end{array}$ & 1 & 1.01 & 1.05 & 1.05 \\
\hline $\begin{array}{c}\text { Computational } \\
\text { Intensity }\end{array}$ & 1 & 0.77 & 0.85 & 1.38 \\
\hline
\end{tabular}

The parallel performance of the code have also been tested and Figure 22 shows the scalability of $O$ penFOAM ${ }^{\circledR}$ on the Opteron blade in use. As it can be seen the code speedup is almost linear with the number of processes employed for the run. To be noted also that the deviation from the ideal trend occurring at the last computational point has to be probably attributed at the pretty small amount of cells $(<50000)$ for each partition thus enhancing inter-process communication time. 


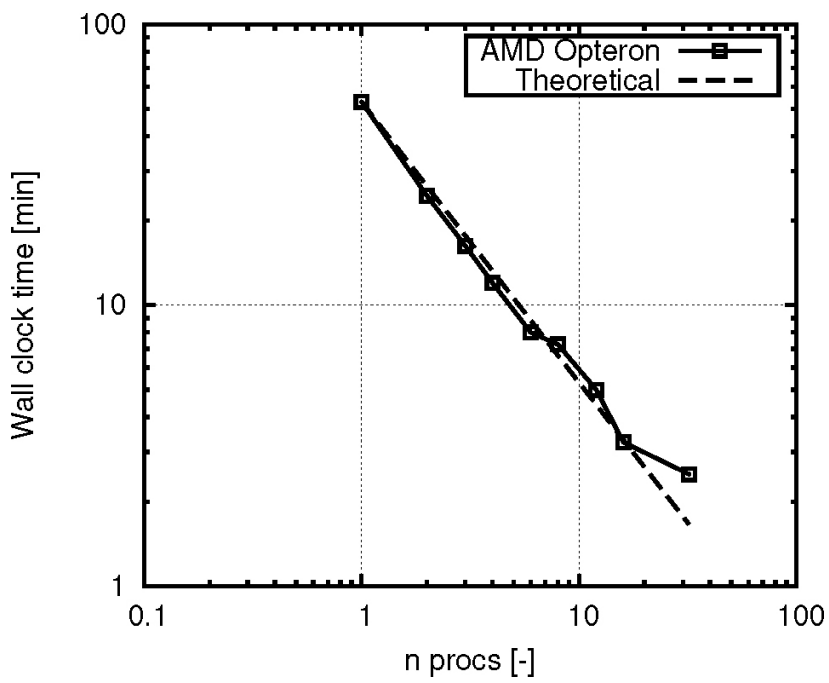

Figure 22: OpenFOAM ${ }^{\circledR}$ scalability (tests performed on the quad-proc. AMD Opteron 6212 blade.

\section{CONCLUSIONS}

The paper presents the implementation and the assessment of two LES SGS turbulence models into the open-source

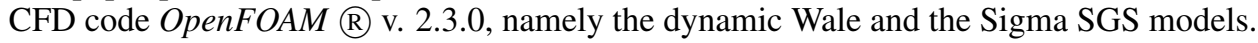

The solver basic capabilities have been extended implementing the aforementioned SGS models whose predictive capabilities have been assessed performing LES analyses on the Dellenback abrupt expansion test case.

A preliminary code assessment phase allowed to evaluate the basic solver suitability for LES analyses by evaluating its numerical dissipation and dispersion tendency. The test regarded an inviscid convection of a vortex in a uniform flow and the outcome led to consider the code suitable for LES analyses, provided that the $C_{o}<0.5$ in order to reduce numerical dissipation.

The abrupt expansion computational results have been compared to the experimental data available and an overall good agreement between the two data sets has been found. The Sigma model performed better than the dynamic Wale and its predictive capabilities are comparable to the Wale model. Both the implemented SGS models outperform the dynamic Smagorinsky model which demonstrated poorer accuracy in wall regions.

Discrepancies between experiments and simulations appear when the models are run on fine grids, in particular at the pipe axis region. In the authors' opinion, the behavior has to be attributed to an improper tuning of the models constants and the issue is currently under investigation.

LES simulations quality has been evaluated through a series of one-grid and two-grid estimators. The main findings are that such estimators present similar distributions for all grids leading to qualitatively comparable indications for all the SGS models tested. The only differences emerge in terms of range of values, sensitivity and easiness of applicability. The medium and fine grid proved to provide sufficient resolution with respect to the purpose of the current investigation with some deficiencies at walls where wall functions have been used.

The computational costs and the memory requirements related to the adoption of the different SGS models have been also estimated. The Sigma model resulted the more demanding model while the dynamic Wale requirements lie in between the ones of the two reference models, i.e. the dynamic Smagorinsky and the Wale models.

In light of the findings presented in this work it can be concluded that the assessed SGS models look promising for applications in LES simulation of ICE flows. However both models require further development in order to overcome some predictive deficiencies. Such developments are currently ongoing and will be the subject of future publications.

\section{REFERENCES}

1. Thobois, L., Rymer, G., SoulÃ̈res, T., and Poinsot, T., "Large-Eddy Simulation in IC Engine Geometries," SAE Technical Paper 2004-01- 1854, 2004, doi:10.4271/2004-01-1854. 
2. Thobois L., Rymer G., Souleres T., Poinsot T., and Van Den Heuvel B. . Large-eddy simulation for the prediction of aerodynamics in ic engines. International Journal of Vehicle Design, 39(4):368-382, January 2005.

3. Brusiani, F., Forte, C., and Bianchi, G., "Assessment of a Numerical Methodology for Large Eddy Simulation of ICE Wall Bounded Non-Reactive Flows," SAE Technical Paper 2007-01-4145, 2007, doi:10.4271/2007-01-4145.

4. Brusiani, F. and Bianchi, G., "LES Simulation of ICE Non-Reactive Flows in Fixed Grids," SAE Technical Paper 2008-01-0959, 2008, doi:10.4271/2008-01-0959.

5. Piscaglia F., Montorfano A., Onorati A., and Brusiani F.. Boundary conditions and sgs models for les of wallbounded separated flows: An application to engine-like geometries. Oil Gas Sci. Technol. - Rev. IFP Energies nouvelles, 69(1):11-27, January 2014.

6. Keskinen, J., Vuorinen, V., and Larmi, M., "Large Eddy Simulation of Flow over a Valve in a Simplified Cylinder Geometry," SAE Technical Paper 2011-01-0843, 2011, doi:10.4271/2011-01-0843.

7. Keskinen, J., Vuorinen, V., Kaario, O., and Larmi, M., "Large Eddy Simulation of the Intake Flow in a Realistic Single Cylinder Configuration,” SAE Technical Paper 2012-01-0137, 2012, doi:10.4271/2012-01-0137.

8. Huijnen V., Olbricht C., Sadiki A., Somers L. M., Baert R. S., Janicka J., and de Goey L. P.. Study of turbulent flow structures of a practical steady engine head flow using large-eddy simulations. Journal of Fluids Engineering, 128(6):1181-1191, April 2006. ISSN 0098-2202.

9. Moureau, V., Barton, I., Angelberger, C., and Poinsot, T., "Towards Large Eddy Simulation in Internal-Combustion Engines: Simulation of a Compressed Tumble Flow," SAE Technical Paper 2004-01-1995, 2004, doi:10.4271/200401-1995.

10. Liu, K. and Haworth, D., "Development and Assessment of POD for Analysis of Turbulent Flow in Piston Engines," SAE Technical Paper 2011-01-0830, 2011, doi:10.4271/2011-01-0830.

11. Benoit Enaux, Victor Granet, Olivier Vermorel, Corine Lacour, Ludovic Thobois, Vincent Dugu, and Thierry Poinsot. Large eddy simulation of a motored single-cylinder piston engine: Numerical strategies and validation. SAE, 86(2):153-177-, 2011. ISSN 1386-6184.

12. Haworth D.C. and Jansen K.. Large-eddy simulation on unstructured deforming meshes: towards reciprocating ic engines. Computers \& Fluids, 29(5):493-524, June 2000. ISSN 0045-7930.

13. Haworth, D. C. Large-eddy simulation of in-cylinder flows. Oil \& Gas Science and Technology - Rev. IFP, 54(2):175-185, 1999. doi: 10.2516/ ogst:1999012.

14. Thobois, L., Lauvergne, R., and Poinsot, T., "Using LES to Investigate Reacting Flow Physics in Engine Design Process,” SAE Technical Paper 2007-01-0166, 2007, doi:10.4271/2007-01-0166.

15. Richard S., Colin O., Vermorel O., Benkenida A., Angelberger C., and Veynante D.. Towards large eddy simulation of combustion in spark ignition engines. Proceedings of the Combustion Institute, 31(2):3059 - 3066, 2007. ISSN 1540-7489. doi: http://dx.doi.org/10.1016/j. proci.2006.07.086.

16. Vermorel O., Richard S., Colin O., Angelberger C., Benkenida A., and Veynante D.. Towards the understanding of cyclic variability in a spark ignited engine using multi-cycle \{LES $\}$. Combustion and Flame, 156(8):1525 - 1541, 2009. ISSN 0010-2180. doi: http://dx.doi . org/10.1016/j.combustflame.2009.04.007.

17. Stephen B Pope. Ten questions concerning the large-eddy simulation of turbulent flows. New Journal of Physics, 6(1):35, 2004.

18. Celik IB, Cehreli ZN, and Yavuz I. Index of resolution quality for large eddy simulations. Journal of Fluids Engineering, 127(5):949-, 2005. ISSN 00982202.

19. Francesca di Mare, Robert Knappstein, and Michael Baumann. Application of les-quality criteria to internal combustion engine flows. Computers \& Fluids, 89(0):200 - 213, 2014. ISSN 0045-7930.

20. Brusiani, F., Bianchi, G., Baritaud, T., and d'Espinosa, A., "Using LES for Predicting High Performance Car Airbox Flow," SAE Int. J. Passeng. Cars - Mech. Syst. 2(1):1050-1064, 2009, doi:10.4271/2009-01-1151.

21. Charles Hirsch, editor. Numerical Computation of Internal and External Flows: Fundamentals of Numerical Discretization. John Wiley \& Sons, Inc., New York, NY, USA, 1988. ISBN 0-471-91762-1.

22. Nicoud F. and Ducros F.. Subgrid-scale stress modelling based on the square of the velocity gradient tensor. Flow, Turbulence and Combustion, 62(3): 183-200, 1999. ISSN 1386-6184. 
23. Abraham, P., Liu, K., Haworth, D., Reuss, D., and Sick, V. Evaluating large-eddy simulation (les) and high-speed particle image velocimetry (piv) with phase-invariant proper orthogonal decomposition (pod). Oil Gas Sci. Technol. Rev. IFP Energies nouvelles, 69(1):41-59, 2014.

24. Plengsaard, C. and Rutland, C., "Improved Engine Wall Models for Large Eddy Simulation (LES)," SAE Technical Paper 2013-01-1097, 2013, doi:10.4271/2013-01-1097.

25. Misdariis, A., Robert, A., Vermorel, O., Richard, S., and Poinsot, T. Numerical methods and turbulence modeling for les of piston engines: Impact on flow motion and combustion. Oil Gas Sci. Technol. - Rev. IFP Energies nouvelles, 69(1):83-105, 2014.

26. BayaToda H., Truffin K., and Nicoud F.. Is the dynamic procedure appropriate for all sgs models? In Poceedings of ECCOMAS CFD 2010. ECCOMAS, 2010.

27. Baya Toda H., Cabrit O., Balarac G., Bose S., Lee J., Choi H., and Nicoud F.. A subgrid-scale model based on singular values for les in complex geometries. In Poceedings of Summer Program 2010. Center for Turbulence Research, 2010.

28. Weller H. G., Tabor G., Jasak H., and Fureby C.. A tensorial approach to computational continuum mechanics using object-oriented techniques. Comput. Phys., 12(6):620-631, 1998. ISSN 0894-1866.

29. OpenFOAM Foundation. OpenFOAM-2.3.0 Programmer's Guide. OpenFOAM Foundation, 2014.

30. Dellenback P. A., Metzger D. E., and Neitzel G. P.. Measurements in turbulent swirling flow through an abrupt axisymmetric expansion. AIAA Journal, 26(6):669-681, June 1988. ISSN 0001-1452. doi: 10.2514/3.9952.

31. Germano Massimo, Piomelli Ugo, Moin Parviz, and Cabot William H.. A dynamic subgrid scale eddy viscosity model. Physics of Fluids A: Fluid Dynamics (1989-1993), 3(7):1760-1765, 1991.

32. Lilly D. K.. A proposed modification of the germano subgrid-scale closure method. Physics of Fluids, 1992.

33. Vreman A. W.. An eddy-viscosity subgrid-scale model for turbulent shear flow: Algebraic theory and applications. Physics of Fluids (1994-present), 16(10):3670-3681, 2004.

34. Jukka-Pekka Keskinen, Ville Vuorinen, Ossi Kaario, Martti Larmi. Effects of mesh deformation on the quality of large eddy simulations. In Fluid Dynamics and Co-located Conferences, pages -. American Institute of Aeronautics and Astronautics, June 2013.

35. SB Pope. Turbulent flows. Cambridge University Press, 2000.

36. Durbin Paul and Pettersson Reif B.A.. Statistical theory and modeling for turbulent flows. Wiley, 2011.

37. Ferziger J.H. and Peric M.. Computational Methods for Fluid Dynamics. Springer Berlin Heidelberg, 2001.

38. Yee H. C., Vinokur M., and Djomehri M. J.. Entropy splitting and numerical dissipation. J. Comput. Phys., 162(1):33-81, July 2000. ISSN 0021-9991. doi: 10.1006/jcph.2000.6517.

39. Parthib, R. Rao and Laura, A. Schaefer. Numerical stability of explicit off-lattice boltzmann schemes: A comparative study. Journal of Computational Physics, 285(0):251-264, March 2015. ISSN 0021-9991.

40. Vuorinen V., Keskinen J.-P., Duwig C., and Boersma B.J.. On the implementation of low-dissipative runge-kutta projection methods for time dependent flows using openfoam. Computers \& Fluids, 93(0):153-163, April 2014. ISSN 0045-7930.

41. Moureau V., Lartigue G., Sommerer Y., Angelberger C., Colin O., and Poinsot T.. Numerical methods for unsteady compressible multi- component reacting flows on fixed and moving grids. Journal of Computational Physics, 202(2):710-736, January 2005. ISSN 0021- 9991.

42. John, B. Heywood. Internal Combustion Engine Fundamentals. Mcgraw-Hill, 1989.

43. John, L. Lumley. Engines: An Introduction. Cambridge University Press, 1999.

44. Kuo, Tang-Wei, Yang, Xiaofeng, Gopalakrishnan, Venkatesh, and Chen, Zhaohui. Large eddy simulation (les) for ic engine flows. Oil Gas Sci. Technol. - Rev. IFP Energies nouvelles, 69(1):61-81, 2014.

45. Petersen, B. and Ghandhi, J., "High Resolution Scalar Dissipation and Turbulence Length Scale Measurements in an Internal Combustion Engine," SAE Int. J. Engines 3(1):65-83, 2010, doi:10.4271/2010-01- 0185.

46. Heim, D. and Ghandhi, J., "A Detailed Study of In-Cylinder Flow and Turbulence using PIV," SAE Int. J. Engines 4(1):1642-1668, 2011, doi:10.4271/2011-01-1287. 
47. Schlter J. U., Pitsch H., and Moin P.. Large-eddy simulation inflow conditions for coupling with reynoldsaveraged flow solvers. AIAA Journal, 42(3):478-484, March 2004. ISSN 0001-1452. doi: 10.2514/1.3488.

48. Ardalan Javadi and Hakan Nilsson. Les and des of strongly swirling turbulent flow through a suddenly expanding circular pipe. Computers \& Fluids, 107: 301-313, 2015. ISSN 0045-7930.

49. Piscaglia, F., Montorfano, A., and Onorati, A., "A Scale Adaptive Filtering Technique for Turbulence Modeling of Unsteady Flows in IC Engines," SAE Int. J. Engines 8(2):426-436, 2015, doi:10.4271/2015-01- 0395.

50. Walter Gyllenram and Hkan Nilsson. Design and validation of a scale- adaptive filtering technique for lrn turbulence modeling of unsteady flow. Journal of Fluid Engineering, Volume 130:10, 2008.

\section{CONTACT INFORMATION}

Mr. Cristian Catellani v.le del Risorgimento, 2 Bologna, 40136, Italy Work phone: 00390512093320 cristian.catellani5@unibo.it

\section{ACKNOWLEDGMENTS}

The research topic of the present paper has been funded by the Italian Govevernement (M.I.U.R) within the funded project PRIN 2010-11 entitled "Intensificazione di Processi Catalitici per Energia Pulita, Trasporti a Basse Emissioni e Chimica Sostenibile usando Schiume a Celle Aperte quali Nuovi Materiali Strutturati Avanzati (FOAMS)"

\section{GLOSSARY}

Co - Courant Number.

GDI - Gasoline Direct Injection.

HCCI - Homogeneous Charge Compression Ignition.

$\boldsymbol{I C E}$ - Internal Combustion Engine.

LDA - Laser-Doppler Anemometry.

LES - Large Eddy Simulation.

$\boldsymbol{L S R}$ - Length Scale Resolution.

$L_{i} j$ - Leonard Stress Tensor.

RANS - Reynolds Averaged Navier Stokes.

$\boldsymbol{R} \boldsymbol{e}$ - Reynolds Number.

$S$ - Swirl Number.

$S G S$ - Sub-Grid Scale.

SPD - Spectral Power Density.

$S \boldsymbol{V} \boldsymbol{S}$ - Shear and Vortex Sensor.

$S_{i j}$ - Resolved Rate-of-strain Tensor.

WALE - Wall-Adapting Local Eddy-viscosity.

$\Delta$ - Filter Width.

$\eta$ - Kolmogorov Microscale Length.

$\nu$ - (Molecular) Kinematic Viscosity.

$\nu_{S G S}$ - Subgrid Eddy Viscosity.

$\rho$ - Density.

$\sigma_{i}$ - Velocity Gradient Tensor Singular Values.

$\tau_{i j}^{S G S}$ - Subgrid Stress Tensor. 
$\epsilon_{S G S}$ - Subgrid Turbulent Dissipation.

$g_{i j}$ - Velocity Gradient Tensor.

$\boldsymbol{k}$ - Turbulent Kinetic Energy.

$k_{S G S}$ - Subgrid Turbulent Kinetic Energy.

$k_{n u m}$ - Numerical Turbulent Kinetic Energy.

$k_{r e s}$ - Resolved Turbulent Kinetic Energy.

$k_{t o t}$ - Total Turbulent Kinetic Energy.

$l_{D I}$ - Inertial Subrange Lower Limit.

$\boldsymbol{p}$ - Static Pressure.

$\boldsymbol{u}$ - Velocity.

All rights reserved. No part of this publication may be reproduced, stored in a retrieval system, or transmitted, in any form or by any means, electronic, mechanical, photocopying, recording, or

otherwise, without the prior written permission of SAE International.

Positions and opinions advanced in this paper are those of the author(s) and not necessarily those of SAE International. The author is solely responsible for the content of the paper. 